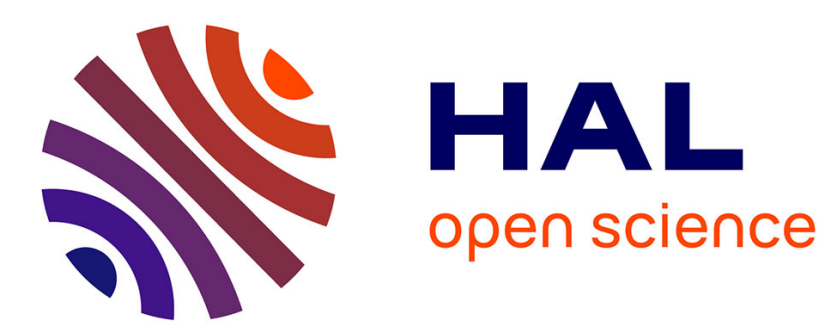

\title{
Data assimilation for identification of cardiovascular network characteristics
}

\author{
Rajnesh Lal, Bijan Mohammadi, Franck Nicoud
}

\section{To cite this version:}

Rajnesh Lal, Bijan Mohammadi, Franck Nicoud. Data assimilation for identification of cardiovascular network characteristics. International Journal for Numerical Methods in Biomedical Engineering, 2017, 33 (5), pp.e2824. 10.1002/cnm.2824 . hal-01359368

\section{HAL Id: hal-01359368 https://hal.science/hal-01359368}

Submitted on 5 Sep 2016

HAL is a multi-disciplinary open access archive for the deposit and dissemination of scientific research documents, whether they are published or not. The documents may come from teaching and research institutions in France or abroad, or from public or private research centers.
L'archive ouverte pluridisciplinaire HAL, est destinée au dépôt et à la diffusion de documents scientifiques de niveau recherche, publiés ou non, émanant des établissements d'enseignement et de recherche français ou étrangers, des laboratoires publics ou privés. 


\title{
Data assimilation for identification of cardiovascular network characteristics
}

\author{
Rajnesh Lal, Bijan Mohammadi, and Franck Nicoud
}

IMAG, Universite de Montpellier, CC051, 34095 Montpellier, France

\begin{abstract}
A method to estimate the hemodynamics parameters of a network of vessels using an Ensemble Kalman filter is presented. The elastic moduli (Young's modulus) of blood vessels and the terminal boundary parameters are estimated as the solution of an inverse problem. Two synthetic test cases and a configuration where experimental data is available are presented. The sensitivity analysis confirms that the proposed method is quite robust even with a few numbers of observations. The simulations with the estimated parameters recovers target pressure or flow rate waveforms at given specific locations, improving the state of the art predictions available in the literature. This shows the effectiveness and the efficiency of both the parameters estimation algorithm and the blood flow model.
\end{abstract}

Keywords: Ensemble Kalman filter, 1D blood flow, parameter estimation, inverse problem.

\section{INTRODUCTION}

An increase in arterial stiffness has been shown to be linked with age including other health problems or risk factors such as diabetes and hypertension [1]. The stiffness of arteries can be measured using different techniques such as by measuring the pulse wave velocity (PWV) or with the analysis of local variations in local pressure and volume [2]. PWV is directly related to the arterial wall elasticity and to the Young's modulus of the arteries [2]. 
"Assimilation is the process of finding the model representation which is most consistent with the observations" [3]. The use of inaccurate parameters in the model equations can give rise to model errors [4]. The parameter estimation problem tends to improve initial estimates of the model parameters so that the difference between the measurements and the model solution are minimised. In parameter estimation problem, it is assumed that the uncertainties in the model parameters are the sources of errors for the model errors [5]. According to [6], it is important to tune the parameters to gain a better confidence in the predictions of the state values. Generally, we have observable data for the state, but no direct observable data for the parameters.

In recent years, parameter estimation has been carried out using a similar framework as for the state estimation. The state vectors can be augmented by the poorly known parameters for estimating by having a Kalman filter for the state-parameter augmented model $[5,7,8,9]$. In state-parameter augmentation, parameters are considered as part of the model, which are updated in the analysis step of the data assimilation algorithm together with the model variables [6]. An evolution model for model parameters is required for the state-parameter augmented model [10]. The common evolution model includes the random walk model $[11,12]$ and the persistence model $[9,13]$. Combining the model variables and model parameters during the analysis step can also introduce problems such as parameter collapse and filter divergence [14]. In [14, 15], parameter estimation using an Ensemble Kalman Filter (EnKF) is presented using augmentation method, but without updating the model states during the assimilation step. 
Some recent works on inverse problems in hemodynamics include the work of Lombardi [16], Moireau et al. [17], Pant et al. [18], Bertoglio et al. [19], Chabiniok et al. [20], Martin et al. [21], Spilker et al. [22] and Lassila et al. [23]. In [16], a sequential approach based on the reduced order unscented Kalman filter (ROUKF) is presented for the identification of arterial stiffness parameters in 1D haemodynamics. In [17], ROUKF is used to identifiy the boundary condition parameters in a fluid structure vascular model utilising patient image data. In [18], a sequential estimation technique using the unscented Kalman filter (UKF) is presented to estimate lumped model parameters from clinical measurements. In [19], parameter estimation using ROUKF for fluid-structure interaction problems is presented. In [20], the use of sequential joint state-parameter data assimilation to a biomechanical heart model with actual cardiac Cine-MRI data are presented. In [21], a variational method (adjoint state approach) is presented to identify the parameters of one-dimensional models for blood flow in arteries. In [22], a quasi-Newton method is used to adjust the parameters of the outlet boundary conditions of blood flow models to achieve target profiles of flow and pressure waveforms. In [23], the solution of inverse problems in hemodynamics is proposed using deterministic and Bayesian approaches.

Recent works on inverse problems in hemodynamics are either based on joint state augmented model (e.g. [20]) or ROUKF (e.g. [16, 17, 18, 19]). We use Ensemble Kalman filters (EnKF) to identify the Young's modulus and the terminal boundary parameters as the solution of inverse problems. Our aim is to show that this can be achieved with only a few number of observations and without using the joint state formulation hence reducing a modification of the state equations. We think this is important in order to minimize the coupling between the assimilation tool 
and the state equations solver. Also, one originality is to ensure positivity for the solutions of the inversion introducing an adequate reformulation of the problem through logarithmic variable changes. Finally, one important result of the paper is to show that joint use of data assimilation and flow solution by a CFD code greatly improves available results in the literature for a realistic human arterial model with available experimental references [24].

In this paper, first, we present a review of the data assimilation method using an Ensemble Kalman filter (EnKF) and propose a method for hemodynamics parameter estimation as the solution to an inverse problem. In the second section, a blood flow model of the cardiovascular network is presented. In section three, test cases are presented where we show the applicability of an Ensemble Kalman filter to 1D blood flow model in parameter estimation. The first two test cases use synthetic data and the final test case involves the use of an experiments data [24]. The test cases are limited to the estimation of Young's modulus, the boundary condition parameter, i.e. reflection coefficient and the viscoelastic coefficient.

\section{Ensemble Kalman Filter}

First introduced by Geir Evenson [25], an Ensemble Kalman Filter (EnKF) solves the Fokker-Plank equation using a Monte Carlo or ensemble integrations [26]. It is a sub-optimal estimator for problems involving high-order non-linear models. The error statistics are predicted using the ensemble of states. Different versions of EnKF are available in the literature: Deterministic Ensemble Kalman filter (DEnKF) [27], Monte Carlo EnKF [25, 28], EnKF [29, 30], Hybrid EnKF [31], Ensemble Transform 
Kalman filter (ETKF) [32], Ensemble Adjustment Kalman filter (EAKF) [7], Ensemble Square Root filters (EnSRF) [33] and Local Ensemble Kalman filter (LEKF) $[34]$.

2.1. Derivation of Ensemble Kalman Filter. In EnKF, the forecast error covariance matrix is evaluated using an ensemble of forecasts. In this section, we follow and describe the different steps employed in the formulation of EnKF as presented in $[30,35,36,37,38]$.

We will assume that the discrete nonlinear system is described by

$$
\mathbf{x}_{k+1}=f\left(\mathbf{x}_{k}\right)+\mathbf{w}_{\mathbf{k}}, \quad \mathbf{y}_{k}=h\left(\mathbf{x}_{k}\right)+\mathbf{v}_{\mathbf{k}} .
$$

The model state at time $t_{k}$ is $\mathbf{x}_{k} \in \mathbb{R}^{n}$, while the observed state is $\mathbf{y}_{k} \in$ $R^{p} . n$ is the dimension of the model state vector and $p$ is the number of observations. $\mathbf{w}_{k} \in$ $R^{n}$ and $\mathbf{v}_{k} \in$ $R^{p}$ are assumed uncorrelated Gaussian model errors with $\mathbf{w}_{k} \sim \mathcal{N}\left(0, \mathbf{Q}_{\mathbf{k}}\right)$ and $\mathbf{v}_{k} \sim$ $\mathcal{N}\left(0, \mathbf{R}_{\mathbf{k}}\right)$ where $\mathbf{Q}_{\mathbf{k}}$ and $\mathbf{R}_{\mathbf{k}}$ are the covariance matrices. $h$ is the function describing the relationship between the measurement and the states.

At time $t_{k}$, it is assumed that an ensemble of $q$ forecast state estimates (prior ensembles $), \mathbf{X}_{k}^{\mathrm{f}}=\left(\mathbf{x}_{k}^{\mathrm{f}_{1}}, \ldots, \mathbf{x}_{k}^{\mathrm{f}_{q}}\right) \in$ $R^{n \times q}$ is available. $\mathrm{f}_{i}$ represents the $i$-th forecast member of the ensemble. The mean of the ensemble of forecast state is $\overline{\mathbf{x}}_{k}^{\mathrm{f}} \in$ 
$R^{n}$ and is given by

$$
\overline{\mathbf{x}}_{k}^{\mathrm{f}}=\frac{1}{q} \sum_{i=1}^{q} \mathbf{x}_{k}^{\mathrm{f}_{i}} .
$$

The forecast covariance matrix, $\mathbf{P}_{k}^{\mathrm{f}} \in$ $R^{n \times n}$ is defined by

$$
\mathbf{P}_{k}^{\mathrm{f}}=\frac{1}{q-1} \sum_{i=1}^{q}\left(\mathbf{x}_{k}^{\mathrm{f}_{i}}-\overline{\mathbf{x}}_{k}^{\mathrm{f}}\right)\left(\mathbf{x}_{k}^{\mathrm{f}_{i}}-\overline{\mathbf{x}}_{k}^{\mathrm{f}}\right)^{\mathrm{T}} .
$$

After the computation of the Kalman gain $\mathbf{K}_{k}$, all operations on the ensemble members are independent in the EnKF analysis step and the ensemble members are updated using:

$$
\mathbf{x}_{k}^{\mathrm{a}_{i}}=\mathbf{x}_{k}^{\mathrm{f}_{i}}+\mathbf{K}_{k}\left[\mathbf{y}_{k}^{i}-h\left(\mathbf{x}_{k}^{\mathrm{f}_{i}}\right)\right], \quad i=1, \ldots, q,
$$

where $\mathrm{a}_{i}$ represents the $i$-th updated or analysed member of the ensemble. Without adding perturbations to the original observation vector, an updated ensemble with a low variance can be obtained [39]. Hence, to maintain the correct forecast error covariance, a suitable spread of the ensemble members is required. This is achieved by using an ensemble of perturbed observations [39]. An ensemble of the same size $q$ consisting of observations is also generated by adding small perturbations to the observation set $\mathbf{y}_{k}$. Perturbations are generated to have the same distribution as the measurement error and the perturbed observations $\mathbf{y}_{k}^{i}$ are defined by

$$
\mathbf{y}_{k}^{i}=\mathbf{y}_{k}+\mathbf{e}_{k}^{i}, \quad i=1, \ldots, q
$$


where $\mathbf{e}_{k}^{i} \in$

$R^{p}$ is a Gaussian random vector with zero mean and a specified variance. The measurement error covariance matrix, $\mathbf{R}_{\mathbf{k}}$, is diagonal following the assumption of independent observations [33] and is defined as

$$
\mathbf{R}_{\mathbf{k}}=\operatorname{diag}\left[\frac{1}{q-1} \mathbf{E E}^{\mathrm{T}}\right], \quad \mathbf{E}=\left[\mathbf{e}_{k}^{1}, \ldots, \mathbf{e}_{k}^{q}\right]
$$

For a linear measurement function, $h$, and if the noise is additive, that is

$$
\mathbf{y}_{k}=\mathbf{H x}_{k}+\mathbf{v}_{\mathbf{k}}
$$

the Kalman gain is defined by [36]

$$
\mathbf{K}_{\mathbf{k}}=\mathbf{P}_{k}^{\mathrm{f}} \mathbf{H}^{\mathrm{T}}\left(\mathbf{H P}_{k}^{\mathrm{f}} \mathbf{H}^{\mathrm{T}}+\mathbf{R}_{k}\right)^{-1}
$$

In Eq. (8), the observation operator, $\mathbf{H} \in$

$R^{p \times n}$ is linear or linearized. To circumvent the linearization of a nonlinear measurement function which might be difficult to linearize, Houtekamer and Mitchell [40] re-wrote the two terms $\mathbf{P}_{k}^{\mathrm{f}} \mathbf{H}^{\mathrm{T}}$ and $\mathbf{H P}_{k}^{\mathrm{f}} \mathbf{H}^{\mathrm{T}}$ which appear in the Kalman gain Eq. (8) as

$$
\begin{aligned}
\mathbf{P}_{k}^{\mathrm{f}} \mathbf{H}^{\mathrm{T}} & \equiv \frac{1}{q-1} \sum_{i=1}^{q}\left[\mathbf{x}_{k}^{\mathrm{f}_{i}}-\overline{\mathbf{x}}_{k}^{\mathrm{f}}\right]\left[h\left(\mathbf{x}_{k}^{\mathrm{f}_{i}}\right)-\overline{h\left(\mathbf{x}_{k}^{\mathrm{f}}\right)}\right]^{\mathrm{T}}, \\
\mathbf{H P}_{k}^{\mathrm{f}} \mathbf{H}^{\mathrm{T}} & \equiv \frac{1}{q-1} \sum_{i=1}^{q}\left[h\left(\mathbf{x}_{k}^{\mathrm{f}_{i}}\right)-\overline{h\left(\mathbf{x}_{k}^{\mathrm{f}}\right)}\right]\left[h\left(\mathbf{x}_{k}^{\mathrm{f}_{i}}\right)-\overline{h\left(\mathbf{x}_{k}^{\mathrm{f}}\right)}\right]^{\mathrm{T}},
\end{aligned}
$$


where $\overline{h\left(\mathbf{x}_{k}^{\mathrm{f}}\right)}=\frac{1}{q} \sum_{i=1}^{q} h\left(\mathbf{x}_{k}^{\mathrm{f}_{i}}\right)$. It has been argued by Tang and Ambadan [41] that Eqs. (9) and (10) approximately hold if the following are true :

$$
\begin{gathered}
\overline{h\left(\mathbf{x}_{k}^{\mathrm{f}}\right)}=h\left(\overline{\mathbf{x}}_{k}^{\mathrm{f}}\right), \\
\operatorname{norm}\left(\mathbf{x}_{k}^{\mathrm{f}_{i}}-\overline{\mathbf{x}}_{k}^{\mathrm{f}}\right) \text { is small for } i=1,2, \ldots q .
\end{gathered}
$$

Equations (9) and (10) linearize the nonlinear function $h$ to $\mathbf{H}$ under the conditions of Eqs. (11) and (12) [37]. For the nonlinear model with a nonlinear measurement function, a general equation for the Kalman gain can be stated as [37]:

$$
\mathbf{K}_{k}=\mathbf{P}_{\mathbf{x y}_{k}}^{\mathrm{f}}\left(\mathbf{P}_{\mathbf{y y}_{k}}^{\mathrm{f}}\right)^{-1}
$$

where the error covariance matrices $\mathbf{P}_{\mathbf{x} \mathbf{y}_{k}}^{\mathrm{f}}$ and $\mathbf{P}_{\mathbf{y} \mathbf{y}_{k}}^{\mathrm{f}}$ are defined as follows:

$$
\begin{aligned}
& \mathbf{P}_{\mathbf{x y}_{k}}^{\mathrm{f}}=\frac{1}{q-1} \sum_{i=1}^{q}\left[\mathbf{x}_{k}^{\mathrm{f}_{i}}-\overline{\mathbf{x}}_{k}^{\mathrm{f}}\right]\left[h\left(\mathbf{x}_{k}^{\mathrm{f}_{i}}\right)-\overline{h\left(\mathbf{x}_{k}^{\mathrm{f}}\right)}\right]^{\mathrm{T}}, \\
& \mathbf{P}_{\mathbf{y y}_{k}}^{\mathrm{f}}=\frac{1}{q-1} \sum_{i=1}^{q}\left[h\left(\mathbf{x}_{k}^{\mathrm{f}_{i}}\right)-\overline{h\left(\mathbf{x}_{k}^{\mathrm{f}}\right)}\right]\left[h\left(\mathbf{x}_{k}^{\mathrm{f}_{i}}\right)-\overline{h\left(\mathbf{x}_{k}^{\mathrm{f}}\right)}\right]^{\mathrm{T}} .
\end{aligned}
$$

We define the true state (or parameter) as the target of an ideal assimilation. The best forecast state estimate is given by the ensemble mean $\overline{\mathbf{x}}_{k}^{\mathrm{f}}$. The error between $\overline{\mathbf{x}}_{k}^{\mathrm{f}}$ and the true state is given by the standard deviation of the ensemble members around $\overline{\mathbf{x}}_{k}^{\mathrm{f}}$. The final step is the forecast step and involves an ensemble of $q$ forecast states for time $t=k+1$ as,

$$
\mathbf{x}_{k+1}^{\mathrm{f}_{i}}=f\left(\mathbf{x}_{k}^{\mathrm{a}_{i}}\right)+\mathbf{w}_{k}^{i}, \quad i=1,2, \ldots q .
$$


2.2. Summary of Ensemble Kalman Filter Algorithm. We now summarize the forecast and the analysis steps of EnKF presented in section 2.1. A schematic description of the EnKF algorithm is shown in Fig. 1. To start the EnKF, we need to generate an ensemble of $q$ forecast estimates of state associated with their random errors. At $t=k-1$, it is assumed that $\mathbf{x}_{k-1}^{\mathrm{f}_{i}}$ for $i=1, \ldots, q$ are available. We let $p$ denote the number of observations. At time $t=k$, we generate a set of realizations of the state vector $\mathbf{X}_{k}=\left(\mathbf{x}_{k}^{1}, \ldots, \mathbf{x}_{k}^{q}\right)$ and denote the corresponding measurements as $\mathbf{Y}_{k}=\left(y_{k}^{1}, \ldots, y_{k}^{q}\right) \in$ $R^{q \times p}$. $\mathbf{Q}$ and $\mathbf{R}$ correspond to the model and observation error covariance matrices, respectively. We write the equations for the EnKF as:

$$
\begin{aligned}
\mathbf{x}_{k}^{\mathrm{f}_{i}} & =f\left(\mathbf{x}_{k-1}^{\mathrm{a}_{i}}\right)+\mathbf{w}_{k-1}^{i}, \quad i=1, \ldots, q, \\
\mathbf{w}_{k}^{i} & \sim \mathcal{N}\left(0, \mathbf{Q}_{k}\right), \\
\mathbf{P}_{\mathbf{x y}_{k}}^{\mathrm{f}} & =\frac{1}{q-1} \sum_{i=1}^{q}\left[\mathbf{x}_{k}^{\mathrm{f}_{i}}-\overline{\mathbf{x}}_{k}^{\mathrm{f}}\right]\left[h\left(\mathbf{x}_{k}^{\mathrm{f}_{i}}\right)-\overline{h\left(\mathbf{x}_{k}^{\mathrm{f}}\right)}\right]^{\mathrm{T}}, \\
\mathbf{P}_{\mathbf{y y}_{k}}^{\mathrm{f}} & =\frac{1}{q-1} \sum_{i=1}^{q}\left[h\left(\mathbf{x}_{k}^{\mathrm{f}_{i}}\right)-\overline{h\left(\mathbf{x}_{k}^{\mathrm{f}}\right)}\right]\left[h\left(\mathbf{x}_{k}^{\mathrm{f}_{i}}\right)-\overline{h\left(\mathbf{x}_{k}^{\mathrm{f}}\right)}\right]^{\mathrm{T}}+\mathbf{R}_{k}, \\
\mathbf{K}_{k} & =\mathbf{P}_{\mathbf{x y}_{k}}^{\mathrm{f}}\left(\mathbf{P}_{\mathbf{y}_{k}}^{\mathrm{f}}\right)^{-1}, \\
\mathbf{y}_{k}^{i} & =\mathbf{y}_{k}+\mathbf{e}_{k}^{i}, i=1, \ldots, q, \\
\mathbf{x}_{k}^{\mathrm{a}_{i}} & =\mathbf{x}_{k}^{\mathrm{f}_{i}}+\mathbf{K}_{k}\left[\mathbf{y}_{k}^{i}-h\left(\mathbf{x}_{k}^{\mathrm{f}_{i}}\right)\right], i=1, \ldots, q .
\end{aligned}
$$

In the above steps, the superscripts 'f' and 'a' denotes the forecast and the analysis steps respectively. EnKF algorithm yields an ensemble of analyses at time $t=k$, which can be cycled in time. 


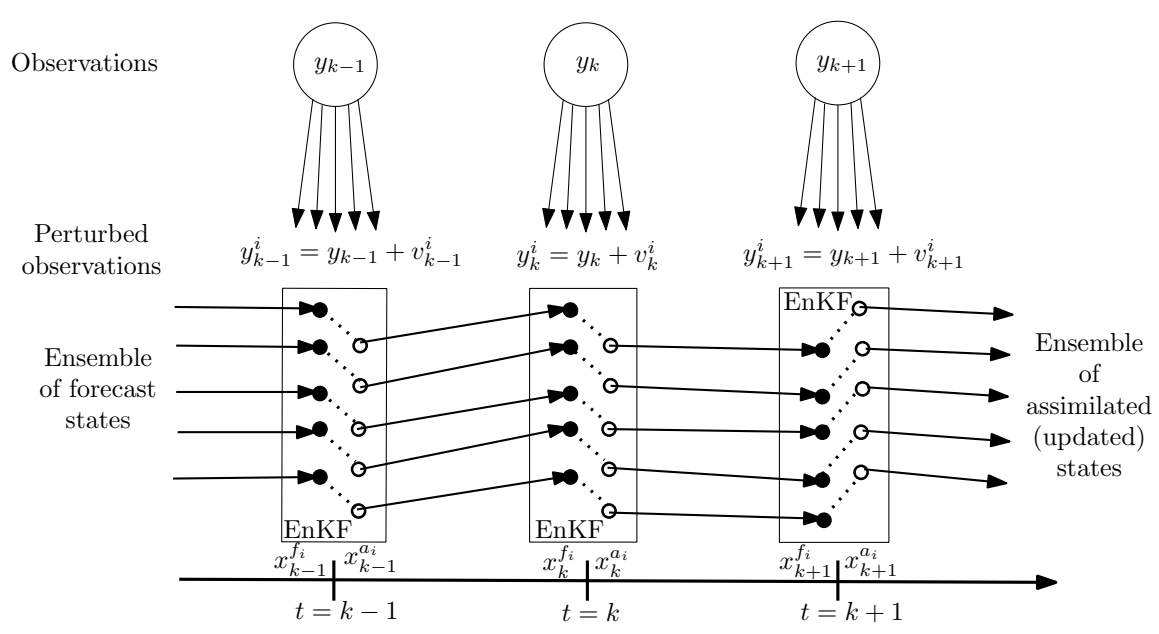

Figure 1. A schematic description of the Ensemble Kalman Filter.

UKF differs from EnKF by the choice of the sampling. UKF uses a minimal set of deterministically chosen points (sigma points) and propagates this set through the actual non-linear function [18]. In contrast, EnKF uses a Monte-Carlo-based choice of many points (members of the ensemble) for forward propagation. UKF should be preferred if the output of the system deviates from a Gaussian distribution. Except with the computation of the Kalman gain, all the operations on the ensemble members are independent. This implies that their parallelization can be trivially carried out. This is one of the reasons for the success and popularity of the EnKF and UKF.

\section{Parameter Estimation Using Ensemble Kalman Filter}

In the current work, the EnKF algorithm is used to estimate only the model parameters. The parameters are thus considered as special state variables (the state vector contains only the model parameters). The evolution of parameters is characterized by a random walk model [11, 12] and is defined as $\mathbf{x}_{k+1}^{i}=\mathbf{x}_{k}^{i}+\tau_{k}^{i}$. $\tau_{k} \sim \mathcal{N}\left(0, \mathbf{T}_{k}\right)$ is a small random perturbation with predefined variance $\mathbf{T}$. Numerical simulators can be regarded as nonlinear functions that take parameter vector 
$\mathbf{x}^{i}$ as an input and produces an output vector $\mathbf{y}^{i}=\mathcal{H}\left(\mathbf{x}^{i}\right)[42] . \mathcal{H}$ is the nonlinear measurement function defined by the numerical simulator. The information from observations are used by the Kalman filter during the analysis step and the Kalman gain Eq. (4) is applied to update the ensemble members. The use of Eq. (4) assumes that the parameters follow a Gaussian distribution [42]. The parameter estimation procedure using the EnKF is stated in Algorithm 1 [42]. The algorithm can be stopped when some finite convergence criterion is achieved. A flowchart for parameter estimation using EnKF is shown in Fig. 2 Positivity issues are physically

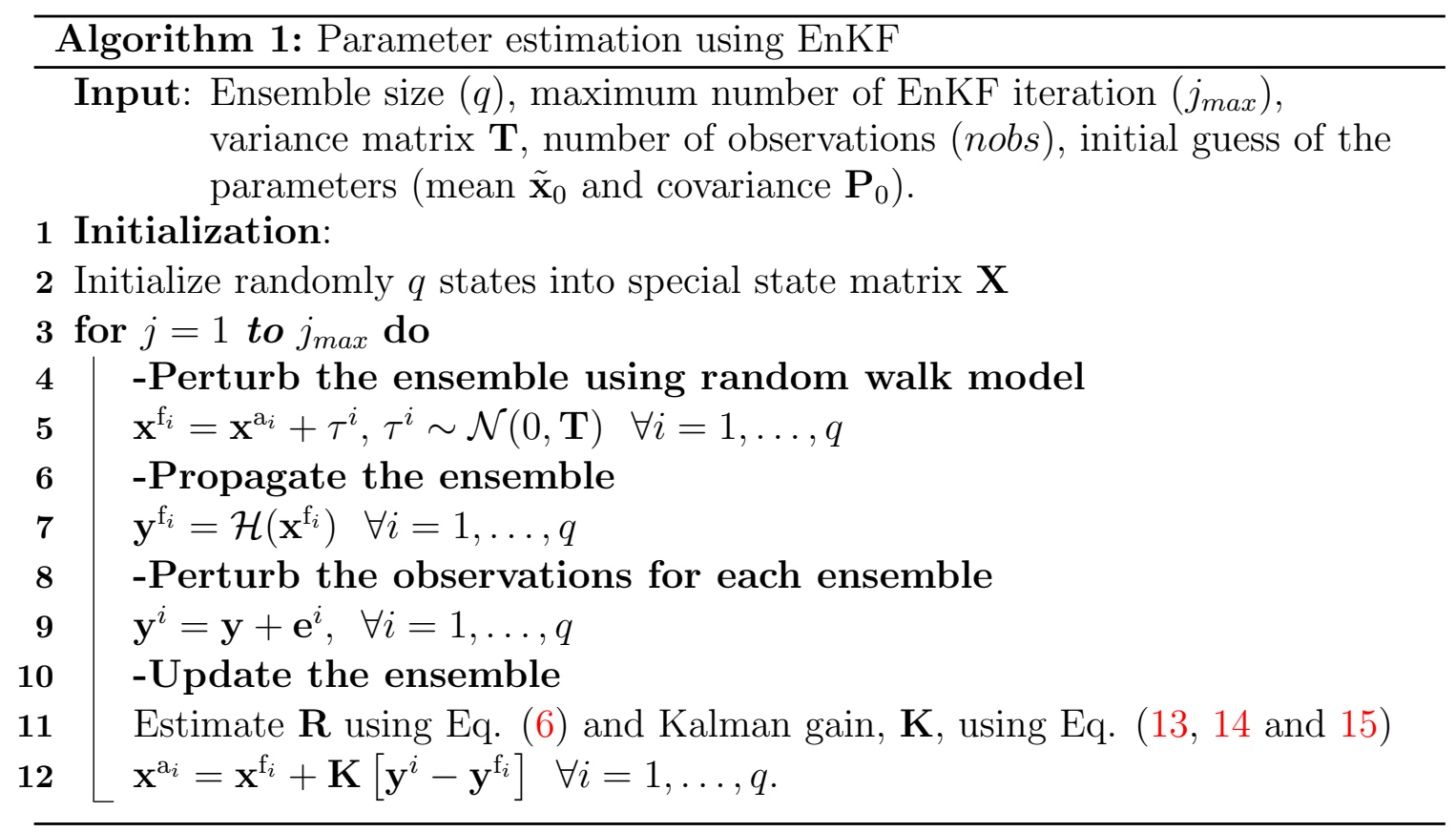

important but often difficult to enforce in assimilation processes. In this study the parameters (e.g. the Young's modulus) need to remain positive. To avoid negative values of the Young's modulus during the assimilation procedure, we introduce a change of variable. More precisely, in the sequel all the parameters are redefined as $x=x_{r e f} 2^{\theta} . x$ is the real parameter (e.g the Young's modulus), $\theta$ is the parameter used for estimation in EnKF and $x_{r e f}$ is a reference value (initial mean value for the Young's modulus). With this change of variable, the values of estimated parameters 


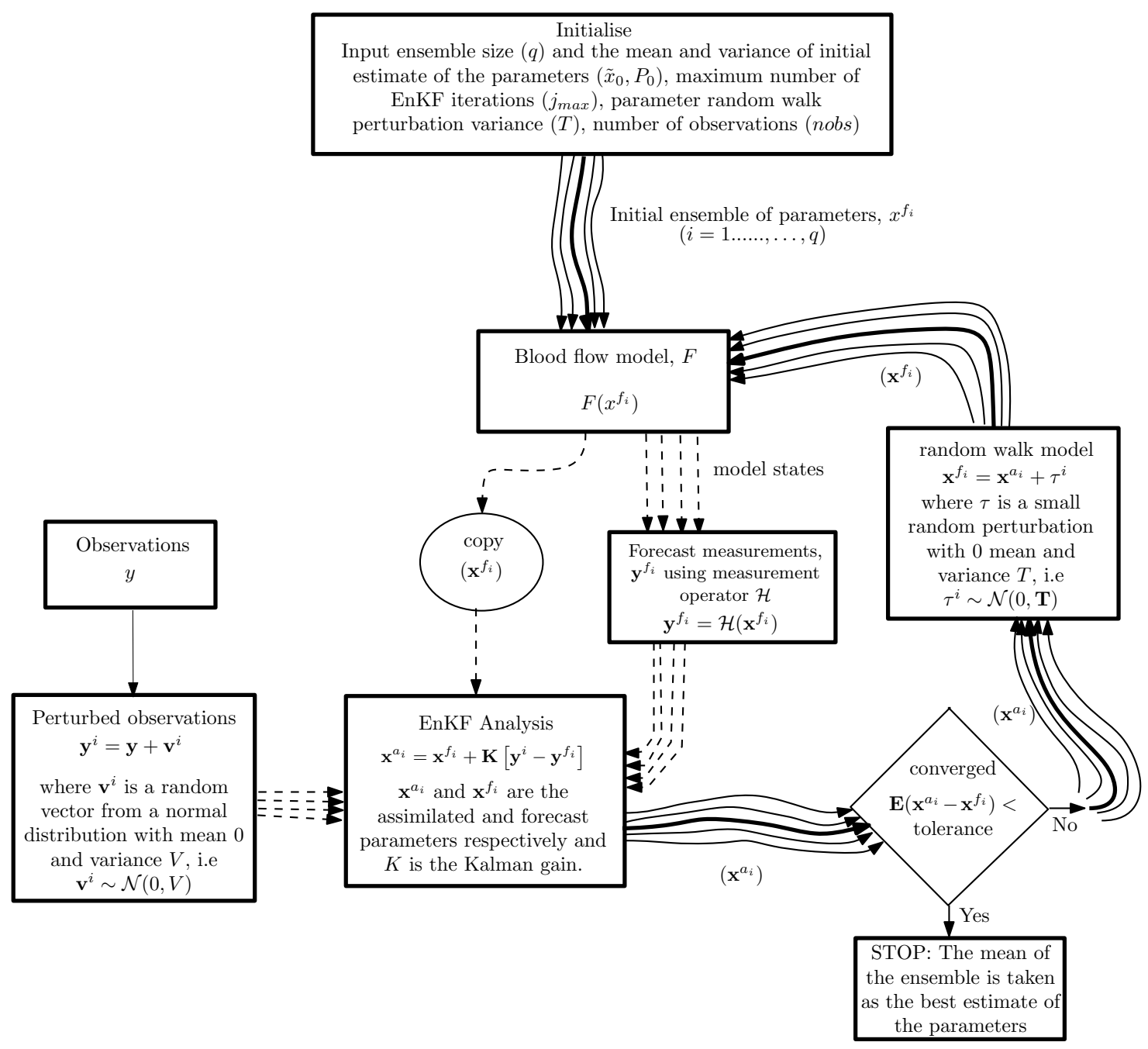

Figure 2. Parameter estimation flowchart using the ensemble Kalman filter.

remain positive [18]. The EnKF implementation uses an ensemble of $\theta$ such that $\theta \sim \mathcal{N}(0,1)$

\section{The Blood Flow Model}

We first recall the one-dimensional (1D) governing equations for the blood flows in variables $u$ (cross section averaged blood velocity), $A$ (cross section area) and $p$ (cross section averaged static pressure), which have been widely used in hemodynamics applications $[24,43,44,45,46,47,48,49,50,51,52]$. 1D modelling of arterial networks being computationally cheap is a common method adopted to perform 
numerical simulations of the hemodynamics in arterial vessels $[47,53]$. The model assumes that blood is a Newtonian fluid in large vessels and can be considered incompressible with constant density $\rho$ and constant dynamic viscosity $\mu$ [50].

4.1. Governing Equations. For an incompressible and Newtonian fluid in an elastic tube, the system of equations that represents continuity of mass and momentum can be stated as [43]:

$$
\begin{aligned}
\frac{\partial A}{\partial t}+\frac{\partial q}{\partial x} & =0 \\
\frac{\partial q}{\partial t}+\frac{\partial}{\partial x}\left(\alpha \frac{q^{2}}{A}\right)+\frac{A}{\rho} \frac{\partial p}{\partial x} & =-k_{r} \frac{q}{A}
\end{aligned}
$$

where $x$ is the axial direction, $A=A(x, t)$ is the cross section area at time $t$, $q=q(x, t)$ is the flow rate across a section, $\rho$ is the constant density of the blood, $p$ is the cross section average static internal pressure and $u(x, t)=\frac{q(x, t)}{A(x, t)}$ denotes the cross section averaged blood velocity. The term $\alpha$ is the momentum-flux correction coefficient. For a flat velocity profile it is assumed that $\alpha=1$ [43]. $k_{r}$ denotes the viscous resistance of the flow per unit length of the tube. $A, q$ and $p$ are the unknowns in the system (17). The system is closed by explicitly providing a differential constitutive pressure-area relationship [43]. A nonlinear model for pressure law is adopted according to Kelvin-Voigt model [24]:

$$
p=p_{\text {ext }}+\frac{\beta}{A_{0}}\left[\left(\sqrt{A}-\sqrt{A_{0}}\right)+\epsilon_{p}\left(\sqrt{A}-\sqrt{A_{0}}\right)^{2}\right]+\frac{\gamma}{A_{0}} \frac{\partial \sqrt{A}}{\partial t}
$$

where $p_{\text {ext }}$ denotes the constant external pressure, $A_{0}=A_{0}(x)$, denotes the vessel sectional area at equilibrium state and $\epsilon_{p}$ is the non linearity coefficient. The term $\gamma$ is $h \eta \sqrt{\pi}$, where $h$ is the thickness of the tube and $\eta$ is the viscoelastic coefficient. 
The coefficient $\beta$, which is related to the arterial stiffness is defined as:

$$
\beta=\frac{\sqrt{\pi} h E}{\left(1-\sigma^{2}\right)},
$$

where $E=E(x)$ is the Young's modulus and $\sigma=0.5$ is the Poisson ratio [43].

With a specified inflow boundary condition, the 1D governing equations for the blood flow are solved using the terminal models for the outflow boundary conditions [47].

4.2. Characteristic variables. The 1D model (17) can be rewritten in a conservative form,

$$
\frac{\partial \mathbf{U}}{\partial t}+\frac{\partial \mathbf{F}(\mathbf{U})}{\partial x}=\mathbf{S}(\mathbf{U})
$$

where $\mathbf{U}=[A, q]^{T}$ denotes the vector of conserved variables, $\mathbf{F}(\mathbf{U})$ are the fluxes and $\mathbf{S}(\mathbf{U})$ are the source terms defined by:

$$
\begin{aligned}
& \mathbf{F}(\mathbf{U})=\left[\begin{array}{c}
q \\
\alpha \frac{q^{2}}{A}+\frac{\beta}{3 \rho A_{0}} A^{\frac{3}{2}}+\frac{\beta \epsilon_{p}}{\rho A_{0}}\left(\frac{1}{2} A^{2}-\frac{2}{3} \sqrt{A_{0}} A^{\frac{3}{2}}\right)
\end{array}\right], \\
& \mathbf{S}(\mathbf{U})=\left[\begin{array}{c}
0 \\
-k_{r} \frac{q}{A}+\frac{\gamma \sqrt{A}}{2 \rho A_{0}}\left(\frac{\partial^{2} q}{\partial x^{2}}-\frac{1}{2 A} \frac{\partial A}{\partial x} \frac{\partial q}{\partial x}\right)
\end{array}\right] .
\end{aligned}
$$

The highly coupled system of non-linear equations (20) is decoupled to implement the numerical solution with the prescribed boundary conditions [46]. The characteristic system is derived by expressing the system of equations (20) in a quasi-linear 
form which can be expressed as [46]:

$$
\frac{\partial \mathbf{U}}{\partial t}+\mathbf{J} \frac{\partial \mathbf{U}}{\partial x}=\mathbf{S}
$$

where the Jacobian reads:

$$
\mathbf{J}(\mathbf{U})=\frac{\partial \mathbf{F}}{\partial \mathbf{U}}=\left[\begin{array}{cc}
0 & 1 \\
-\alpha \frac{q^{2}}{A^{2}}+\frac{\beta}{2 \rho A_{0}} A^{\frac{1}{2}}+\frac{\beta \epsilon_{p}}{\rho A_{0}}\left(A-\sqrt{A_{0}} \sqrt{A}\right) & 2 \alpha \frac{q}{A}
\end{array}\right]
$$

By considering the non-linear coefficient $\left(\epsilon_{p}\right)$ and visco elasticity $(\eta)$ as source terms, the characteristic analysis shows that for all allowable $\mathbf{U}$ (that is for $A>0$ ), the system is hyperbolic and the two real eigenvalues of $\mathbf{J}$ are $[48,49]$ :

$$
\begin{aligned}
& \lambda_{1}=\frac{\alpha q}{A}+\sqrt{\frac{\beta}{2 \rho A_{0}} A^{\frac{1}{2}}+\alpha(\alpha-1) \frac{q^{2}}{A^{2}}}>0, \\
& \lambda_{2}=\frac{\alpha q}{A}-\sqrt{\frac{\beta}{2 \rho A_{0}} A^{\frac{1}{2}}+\alpha(\alpha-1) \frac{q^{2}}{A^{2}}}<0 .
\end{aligned}
$$

When $\alpha=1$, the associated characteristic variables have the following expressions:

$$
\begin{aligned}
& W_{1}=\frac{q}{A}+4\left(c-c_{0}\right), \\
& W_{2}=\frac{q}{A}-4\left(c-c_{0}\right),
\end{aligned}
$$

where $c=\sqrt{\frac{\beta}{2 \rho A_{0}}} A^{\frac{1}{4}}$ and $c_{0}=\sqrt{\frac{\beta}{2 \rho A_{0}}} A_{0}^{\frac{1}{4}}$. The characteristic system can be expressed as the decoupled system of equations:

$$
\begin{aligned}
& \frac{\partial w_{1}}{\partial t}+\lambda_{1} \frac{\partial w_{1}}{\partial x}=0 \\
& \frac{\partial w_{2}}{\partial t}+\lambda_{2} \frac{\partial w_{2}}{\partial x}=0 .
\end{aligned}
$$


The wave velocity which arises from the blood wall coupling may take values as low as $5 \mathrm{~m} / \mathrm{s}$ in large arteries (e.g. aorta), increasing to values around $20-35 \mathrm{~m} / \mathrm{s}$ in less distensible peripheral arteries [50]. However, peak flow velocities $u$ are much smaller and usually remain less than around $1 \mathrm{~m} / \mathrm{s}$.

4.3. Numerical approximation. Several methods have been used, e.g. in [43, $44,45,52,54]$, for the numerical approximation of the 1D system of conservation laws (17). In this section, we follow $[45,54]$ where equations of the $1 \mathrm{D}$ model are discretized in their conservative form (20) by employing a second order Taylor Galerkin scheme. We denote $\triangle t=t^{n+1}-t^{n}$ the time step and express the Taylor expansion truncated to the second order at time $t^{n}$, giving

$$
\mathbf{U}^{n+1}=\mathbf{U}^{n}+\left.\triangle t \frac{\partial \mathbf{U}}{\partial t}\right|^{n}+\left.\frac{\triangle t^{2}}{2} \frac{\partial^{2} \mathbf{U}}{\partial t^{2}}\right|^{n}
$$

We define the matrix

$$
\mathbf{K}=\frac{\partial \mathbf{S}}{\partial \mathbf{U}}=\left[\begin{array}{cc}
0 & 0 \\
k_{r} \frac{q}{A^{2}}+\frac{\gamma}{4 \rho A_{0}} \frac{1}{\sqrt{A}}\left(\frac{\partial^{2} q}{\partial x^{2}}+\frac{1}{2 A} \frac{\partial A}{\partial x} \frac{\partial q}{\partial x}\right) & -\frac{k_{r}}{A}
\end{array}\right]
$$

and rewrite (20) as

$$
\frac{\partial \mathbf{U}}{\partial t}=\mathbf{S}-\frac{\partial \mathbf{F}}{\partial x}
$$


Using the matrices (23) and (28), we obtain

$$
\begin{aligned}
\frac{\partial^{2} \mathbf{U}}{\partial t^{2}} & =\frac{\partial \mathbf{S}}{\partial \mathbf{U}} \frac{\partial \mathbf{U}}{\partial t}-\frac{\partial}{\partial x}\left(\frac{\partial \mathbf{F}}{\partial \mathbf{U}} \frac{\partial \mathbf{U}}{\partial \mathbf{t}}\right) \\
& =\mathbf{K} \frac{\partial \mathbf{U}}{\partial \mathbf{t}}-\frac{\partial}{\partial x}\left(\mathbf{J} \frac{\partial \mathbf{U}}{\partial \mathbf{t}}\right) \\
& =\mathbf{K}\left(\mathbf{S}-\frac{\partial \mathbf{F}}{\partial x}\right)-\frac{\partial(\mathbf{J S})}{\partial x}+\frac{\partial}{\partial x}\left(\mathbf{J} \frac{\partial \mathbf{F}}{\partial \mathbf{x}}\right)
\end{aligned}
$$

At time $t^{n}=n \triangle t$, the vector of unknowns $\mathbf{U}^{n}$ satisfies the following time marching scheme:

$$
\begin{aligned}
\mathbf{U}^{n+1} & =\mathbf{U}^{n}+\Delta t\left(\mathbf{S}^{n}-\frac{\partial \mathbf{F}^{n}}{\partial x}\right) \\
& +\frac{\triangle t^{2}}{2}\left(\mathbf{K}^{n}\left(\mathbf{S}^{n}-\frac{\partial \mathbf{F}^{n}}{\partial x}\right)-\frac{\partial\left(\mathbf{J}^{n} \mathbf{S}^{n}\right)}{\partial x}+\frac{\partial}{\partial x}\left(\mathbf{J}^{n} \frac{\partial \mathbf{F}^{n}}{\partial x}\right)\right) .
\end{aligned}
$$

The spatial discretization uses linear finite elements. The domain $\Omega$ is subdivided into $N_{e l}$ finite elements $\Omega_{e}$ of size $h_{e}$. We let $V_{h}$ be the set of continuous vector functions in $\Omega$, linear on each element and $V_{h}^{0}$ the subspace of $V_{h}$ whose functions are zero at the endpoints [45]. The solution of (31) requires, for $n \geq 0$, to determine $\mathbf{U}^{n+1}$ in $V_{h}$ such that $\forall \phi_{h} \in V_{h}^{0}$,

$$
\begin{aligned}
\left(\mathbf{U}^{n+1}, \phi_{h}\right) & =\left(\mathbf{U}^{n}, \phi_{h}\right)+\triangle t\left(\mathbf{S}^{n}, \phi_{h}\right)+\triangle t\left(\mathbf{F}^{n}, \frac{\partial \phi_{h}}{\partial x}\right) \\
& +\frac{\triangle t^{2}}{2}\left(\mathbf{K}^{n}\left(\mathbf{S}^{n}-\frac{\partial \mathbf{F}^{n}}{\partial x}\right), \phi_{h}\right) \\
& +\frac{\triangle t^{2}}{2}\left(\mathbf{J}^{n}\left(\mathbf{S}^{n}-\frac{\partial \mathbf{F}^{n}}{\partial x}\right), \frac{\partial \phi_{h}}{\partial x}\right),
\end{aligned}
$$

where $(\mathbf{U}, \phi)=\int_{0}^{L} \mathbf{U} \cdot \phi d x$. 
For the stability of the numerical method, we follow [54] and impose the following limitation for the time step:

$$
\triangle t \leq \mathrm{CFL} \times \min _{0 \leq i \leq N_{e l}}\left[\frac{h_{i}}{\max \left(\lambda_{1, i} \lambda_{1, i+1}\right)}\right]
$$

where $\lambda_{1, i}$ is the value of $\lambda_{1}$ at mesh node $x_{i}$ and the maximum CFL number is $\frac{\sqrt{3}}{3}$ [54].

4.4. Initial and boundary conditions. The initial conditions for (31) are given by:

$$
A(x, 0)=A_{0}(x), q(x, 0)=0, p(x, 0)=p_{0}(x),
$$

where $A_{0}(x)$ and $p_{0}(x)$ are the prescribed functions. The hyperbolic nature of the system permits to impose the flow rate $q$ or area $A$ at the inlet [43]. At the inlet usually, the flow rate is specified [55],

$$
q(0, t)=q_{\text {in }}(t)
$$

Information from the outside and inside of the domain are carried by the incoming characteristic $\left(W_{1}\right)$ and the outgoing characteristics $\left(W_{2}\right)$ respectively [44].

At each end of the tube, a single boundary condition is implemented. This is due to the characteristic analysis and using the fact that the flow is subcritical (the eigenvalues $\left(\lambda_{1}\right.$ and $\left.\lambda_{2}\right)$ in (24) have opposite signs) under physiological conditions $[43,51]$. At the inlet at $x=0, \mathbf{U}^{n}$ is assumed to be known and $\lambda_{2}$ in (26) is linearised by taking its value at time $t^{n}$. It can be shown that at the time $t^{n+1}$, the solution 
corresponding to this linearised problem yields $[43,46]$

$$
W_{2}^{n+1}(0)=W_{2}^{n}\left(-\lambda_{2}^{n}(0) \triangle t\right)
$$

Equation (36) is a first order extrapolation of $W_{2}$ from the previous time step. Similar treatment at the outlet $x=L$, leads to:

$$
W_{1}^{n+1}(L)=W_{1}^{n}\left(L-\lambda_{1}^{n}(L) \triangle t\right)
$$

4.5. Terminal vessels. To reduce the complexity of the blood flow simulation, smaller arteries, which are downstream of the truncation points are not explicitly accounted for but their effect is represented by proper outflow boundary conditions [56]. The two most common models used are the constant resistance model [24, 44, $46,56,57]$ and the Windkessel model $[52,56,57]$, that can be obtained using an analogy based on electric circuit components.

The constant resistance (CR) model (see Fig. 3) is represented by a resistor $R_{t}$ [56] where it is assumed that the blood pressure, $p(t)-p_{0}$, is proportional to the blood flow rate $q(t)$. The relation between the blood pressure and the flow is given by $p(t)-p_{0}=R_{t} q(t)$, where $R_{t}$ represents the terminal reflection coefficient. The terminal reflection coefficient for a wavefront travelling in the $+x$ direction can be defined in terms of the incoming and outgoing characteristics as [44, 46]

$$
R_{t}=-\frac{\triangle W_{2}}{\triangle W_{1}}=-\frac{W_{2}^{n+1}-W_{2}^{0}}{W_{1}^{n+1}-W_{1}^{0}} .
$$


The values for $W_{1}^{n+1}$ are determined using Eq. (37), whereas $W_{1}^{0}$ and $W_{2}^{0}$ are the initial values [46]. The unknown $W_{2}^{n+1}$ is determined by rearranging Eq. (38), giving

$$
W_{2}^{n+1}=W_{2}^{0}-R_{t}\left(W_{1}^{n+1}-W_{1}^{0}\right)
$$

A reflection coefficient of $R_{t}=0$ represents a non-reflecting boundary condition.

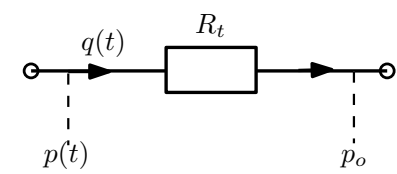

FiguRE 3. A constant resistance (CR) model representing an outflow boundary condition.

4.6. Treatment of bifurcations. At bifurcation of a blood vessel, we assume that pressure losses are negligible. We follow [44] and enforce the following conditions:

$$
\begin{aligned}
\sum_{i=1}^{3} q_{i} & =0, \\
\frac{1}{2} \rho\left(\frac{q_{1}}{A_{1}}\right)^{2}+p_{1}-\frac{1}{2} \rho\left(\frac{q_{i}}{A_{i}}\right)^{2}-p_{i} & =0, \quad i=1,2
\end{aligned}
$$

representing the conservation of flow rate and total pressure continuity equations respectively.

\section{Application of EnKF to 1D Blood flow MOdel}

In this section, we present the use of the EnKF algorithm to solve the parameter estimation problem in a series of test cases. The first two tests are purely in silico, i.e. we use only synthetic data (observations) which are obtained from a forward simulation where the model parameters are set to some known or target values. From these observations, the parameter estimation problem then starts with an initial estimate for the parameters that differs significantly from the target values. 
With synthetic data, an inverse problem is always admissible when the objective is to recover the target parameters. By admissible we mean that because the target is generated with the model, the solution of the inversion targeting this results obviously exists, but still there is no guarantee of uniqueness. Indeed, regardless of existence of solution which is guaranteed in this case, several distributions of the parameters could achieve the target. The final test case uses data from the experiment performed by Saito et al. [24]. In this latter case, unlike with the synthetic data, there is no guarantee that the solution to the inverse problem actually exists.

In [24], a simple human arterial network was designed using polymer tubes to validate the applicability of the $1 \mathrm{D}$ blood flow model. The network was made with four bifurcations and consisted of the main artery, a left carotid artery, femoral arteries (left and right), and subclavian-radial arteries (left and right). The schematic of this simple human arterial model is shown in Fig. 4 and the geometry of the arteries (length, diameter and the thickness) in Table 1 [24]. An appropriate length of blood vessels was defined according to the vessel data of an average adult man. The diameter and thickness were defined to achieve a negligible reflection coefficient at bifurcation point [24]. For all test cases, we use the simple arterial model as in Fig. 4. We limit our parameters of interest to Young's modulus and terminal model parameters (reflection coefficient), which are within a physiological range.

5.1. Two test cases with synthetic data. One of the important parameters for EnKF is q, the ensemble size. It is expected that the EnKF parameter estimation procedure would improve as the ensemble size increases. The increase in an ensemble size will also increase the computational cost associated with it. In our test cases, 


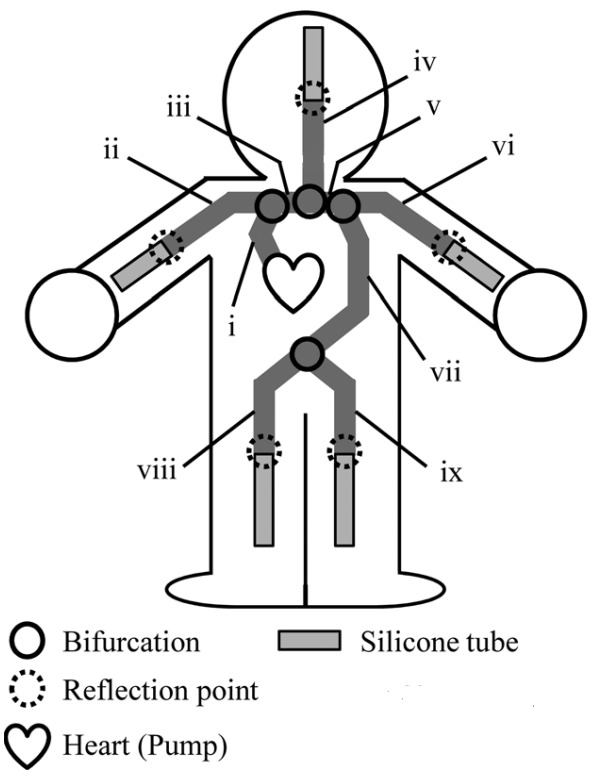

FIgURE 4. Schematic of a simple human arterial model with nine vessels and four bifurcations. Artery numbers corresponds to those in Table 1. Figure adapted from [24].

TABLE 1. Geometrical data ( $L=$ length, $D=$ diameter and $h=$ thickness) of a simple human arterial model (Fig. 4) [24].

\begin{tabular}{|c|l|c|c|c|}
\hline & Name & $\begin{array}{c}L \\
(\mathrm{~mm})\end{array}$ & $\begin{array}{c}D \\
(\mathrm{~mm})\end{array}$ & $\begin{array}{c}h \\
(\mathrm{~mm})\end{array}$ \\
\hline i & $\begin{array}{l}\text { Aorta arch } \\
\text { A }\end{array}$ & 35 & 12 & 2 \\
\hline ii & $\begin{array}{l}\text { R.subclavian } \\
\text { radial } \\
\text { artery }\end{array}$ & 800 & 6 & 1.5 \\
\hline iii & $\begin{array}{l}\text { Aorta arch } \\
\text { B }\end{array}$ & 20 & 11 & 2 \\
\hline iv & $\begin{array}{l}\text { L.carotid } \\
\text { artery }\end{array}$ & 675 & 6 & 1.5 \\
\hline v & $\begin{array}{l}\text { Aorta arch } \\
\text { C }\end{array}$ & 40 & 10 & 2 \\
\hline vi & $\begin{array}{l}\text { L.Subclavian } \\
\text { radial } \\
\text { artery }\end{array}$ & 710 & 6 & 1.5 \\
\hline vii & Aorta & 470 & 8 & 1.5 \\
\hline viii & $\begin{array}{l}\text { R.femoral } \\
\text { artery }\end{array}$ & 365 & 6 & 1.5 \\
\hline ix & $\begin{array}{l}\text { L.femoral } \\
\text { artery }\end{array}$ & 365 & 6 & 1.5 \\
\hline
\end{tabular}


we assume that the blood flow model errors and uncertainties arise from the errors in the parameters and thus an ensemble is generated with perturbed parameters. As detailed in what follows, we therefore performed a parametric study to select a proper ensemble size.

We first simulate the blood flow model using an arbitrary set of parameters $\left\{E, R_{t}\right\}$. The resulting simulated model states are stored as $p^{\text {sim }} \in$ $R^{n}$. An ensemble of size $q$ is generated where the ensemble members are $\left\{E^{\prime}, R_{t}^{\prime}\right\}_{i}$ for $i=1 \ldots, q$. For each $i, E^{\prime}$ is a random normal variable with mean $E$ and standard deviation of $0.1 E$. Similarly, $R_{t}^{\prime}$ is a random normal variable with mean $R_{t}$ and standard deviation of $0.1 R_{t}$. The blood flow model is then simulated with each member of the ensemble and the observed pressure values at the end of the simulation are stored as $p^{o b s_{i}} \in$ $R^{n}$. To select an ensemble size for the EnKF analysis, we calculated the root mean square error defined for each member of the ensemble as: $\operatorname{RMSE}_{i}=\sqrt{\frac{1}{n} \sum_{j=1}^{n}\left(p_{j}^{s i m}-p_{j}^{o b s_{i}}\right)^{2}}$. Finally, we find the mean RMSE for the ensemble of size $q$ as: RMSE $=\frac{1}{q} \sum_{i=1}^{q} \mathrm{RMSE}_{i}$. The procedure was repeated with different ensemble sizes between $q=2$ and $q=60$. From Figure 5 which shows the output of the procedure with different random seeds, the mean RMSE decreases sharply initially with $q$ increasing. From this figure, the error does not decrease after $q \approx 20$ and this latter value was thus retained in the present study.

5.1.1. Test case 1: The first test case deals with the estimation of the Young's modulus for a single artery. We first describe the procedure for generating the synthetic data. All arteries except Aorta (number vii in Fig. 4) is assigned a Young's modulus 


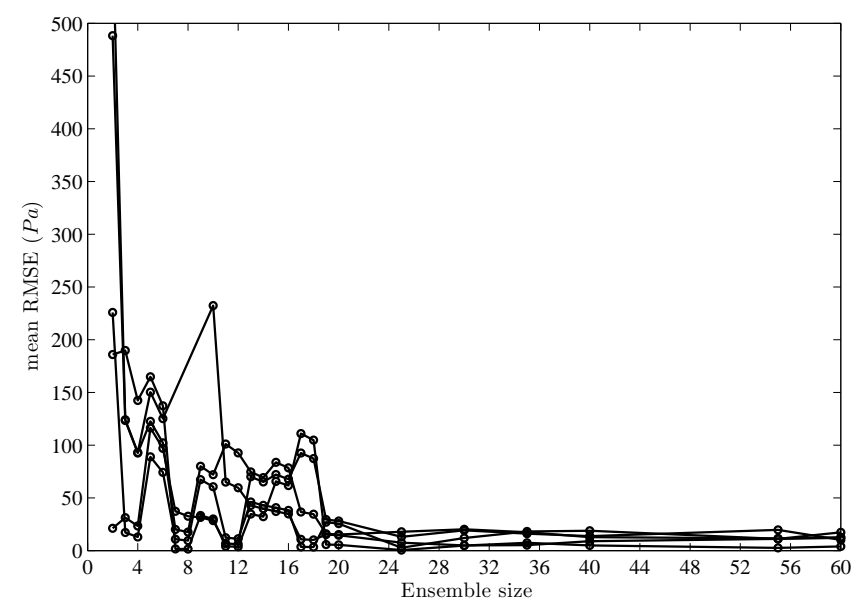

FiguRE 5. Mean RMSE as a function of ensemble size. Five sets of RMSE are calculated with different random seeds.

of 0.2 MPa and to Aorta (number vii), we assigned 0.25 MPa assuming some pathology there locally increasing its stiffness. A CR model is applied to the terminal vessels. A reflection coefficient of $R_{t}=0.6$ is assigned to the terminal vessels ii, iv and vi and for terminal vessels vii and ix, $R_{t}=0.65$. Figure 6 shows the periodic inlet flow rate boundary condition $q_{\text {in }}(t)$ (with a period of $0.8 \mathrm{~s}$ and an average inlet flow of $5.625 \mathrm{ml} / \mathrm{s}$ ) imposed at the aorta arch $\mathrm{A}$ of the simple arterial model. The density of the fluid is taken as $1.0 \mathrm{~kg} / \mathrm{m}^{3}$, the viscosity of $1 \times 10^{-3}$ Pa.s and the Poisson coefficient is taken as 0.5 . The viscoelastic coefficient $\eta$ and the non-linearity coefficient $\epsilon_{p}$ of the vessel are set to 0 for the forward simulation. The time step for the forward simulation is $0.1 \mathrm{~ms}$, corresponding to $C F L=0.05$. Synthetic pressure observations are taken at every $0.01 \mathrm{~s}$. The first objective is to determine the minimum number of observations (nobs), needed for a proper parameter estimation. nobs refers to the number of locations where a time series of pressure is available. Other time series can be considered and we will present also simulations with flow rate time series in section 5.1.2. Algorithm 1 is executed initially with nobs $=6$; nobs is then decreased in steps of one to a minimum of 1 . These observations are 


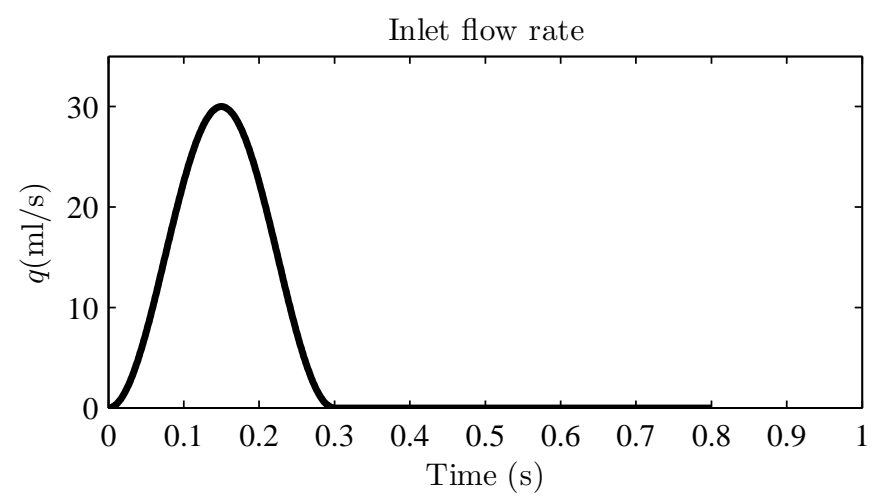

Figure 6. Periodic inlet flow rate $q_{\text {in }}(t)$ imposed at the aorta arch A.

assumed to be available from the left and right subclavian radial arteries (artery \# ii and vi) and the left carotid artery (artery \# iv). The locations of these observations are shown in Table 2.

TABLE 2. Location of observations on right subclavian radial artery (ii), left carotid artery (iv) and left subclavian artery (vi) for synthetic test cases. $L$ is the length of the artery.

\begin{tabular}{|c|c|c|c|c|c|c|}
\hline Artery \# & nobs $=1$ & nobs $=2$ & nobs $=3$ & nobs $=4$ & nobs $=5$ & nobs $=6$ \\
\hline ii & & $0.25 L$ & $0.25 L$ & $0.25 L$ & $0.25 L, 0.75 L$ & $0.25 L, 0.75 L$ \\
\hline iv & $0.2 L$ & $0.2 L$ & $0.2 L$ & $0.2 L, 0.8 L$ & $0.2 L$ & $0.2 L, 0.8 L$ \\
\hline vi & & & $0.33 L$ & $0.33 L$ & $0.33 L, 0.67 L$ & $0.33 L, 0.67 L$ \\
\hline
\end{tabular}

For the estimation problem, the Young's modulus is sought for the stiffest aorta, denoted by vii in Fig. 4. An ensemble of $q=20$ members is considered in all the cases. For each member of the ensemble, the observations are perturbed by a random vector drawn from the zero mean Gaussian distribution with a standard deviation at $5 \%$ of the observation value. We compute the measurement error covariance matrix R using Eq. (6). The initial guess for the Young's modulus assumes an error of $100 \%$, i.e. initial mean value for $E=0.5 \mathrm{MPa}$.

The parameter estimation using Algorithm 1 is then performed with different numbers of observations, nobs. The EnKF assimilation is executed for $10 \mathrm{~s}$ and the evolution of the estimated Young's modulus are shown in Fig. 7a. It appears that 
convergence only takes place after some time. This kind of trend is often observed in optimization, especially with methods involving a learning feature. Indeed, there is no guarantee that the first search iterations are performed in a direction (in parameter space) pointing towards a minimum of the error. This behaviour also comes from the fact that the method is by nature explicit, as in a gradient based methods. Table 3 shows the value of estimated Youngs's modulus with errors (percentage deviation from target value) using different number of observations. The percentage deviations from target $E$ were all less than $5 \%$. For this test case, a minimum of 1 observation was enough to recover the Young's modulus requested in the given interval of time. In Fig 7b, the pressure solutions obtained by using the estimated Young's modulus at the first observation point on left carotid are compared with the target and the initial guessed pressure profiles. The comparison is shown for Young's modulus estimated using nobs $=2$. Even though the solution in the vessel whose Young's modulus is sought for is not directly observed, the simulated pressure waveforms are similar in shape to the target pressure waveform with an error of less than $0.2 \%$ in the maximum pressure.

TABLE 3. Test case1: Estimated Youngs's modulus and corresponding errors (percentage deviation from target value) using different number of observations

\begin{tabular}{|c|c|c|c|c|c|c|}
\hline & nobs $=1$ & nobs $=2$ & nobs $=3$ & nobs $=4$ & nobs $=5$ & nobs $=6$ \\
\hline Estimated $E(\mathrm{MPa})$ & 0.239 & 0.245 & 0.243 & 0.245 & 0.241 & 0.246 \\
\hline \% deviation from target $E$ & -4.49 & -1.95 & -2.64 & -1.82 & -3.52 & -1.79 \\
\hline
\end{tabular}

5.1.2. Sensitivity Analysis: In this section, we look at the sensitivity of the parameter estimation algorithm for test case 1 with respect to the following items: (i) initial 


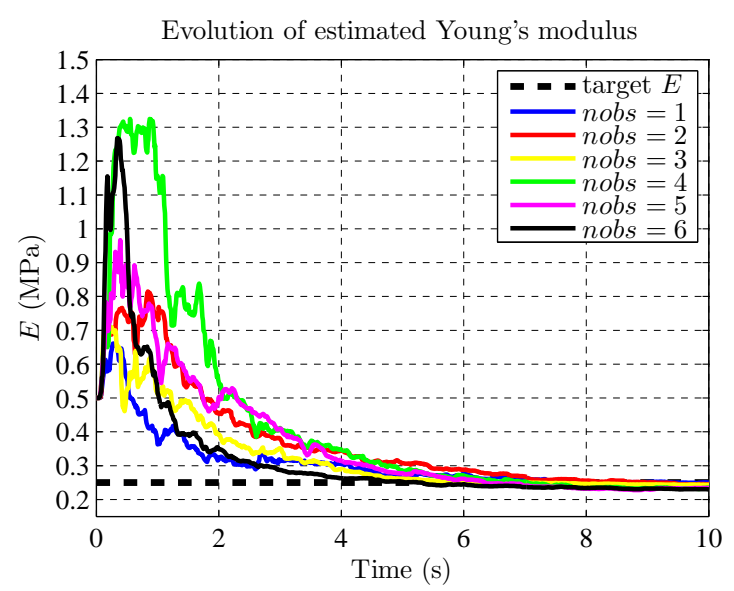

(A)

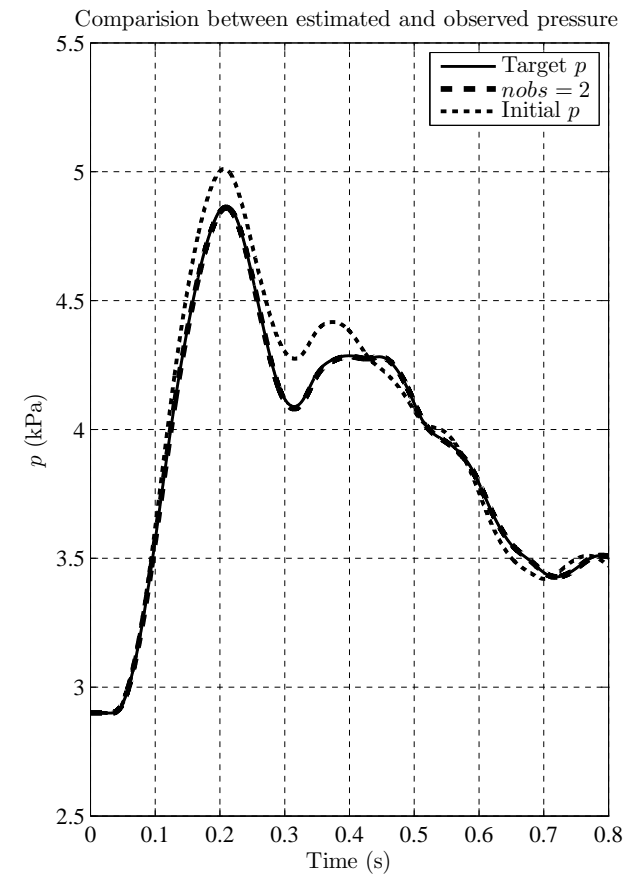

(B)

Figure 7. (7a) shows the evolution of the estimated Young's modulus using EnKF for test case 1 using a different number of observations. The initial value is $0.5 \mathrm{MPa}$ and the target is $0.25 \mathrm{MPa}$. (7b) shows the comparison between target pressure solution, the initial pressure profile and the one obtained by using the estimated Young's modulus with $n o b s=2$ at $0.2 \mathrm{~L}$ of the left carotid artery.

estimate of the parameter, (ii) level of observation perturbation, (iii) the effect of introducing bias in the known parameter values and (iv) the observation type.

(i) Initial guess: we study the performance of EnKF by considering different initial values of the Youngs's modulus for the parameter estimation problem. Two 
more initial values of $E=0.15 \mathrm{MPa}(-40 \%$ error $)$ and $0.35 \mathrm{MPa}(+40 \%$ error $)$ were taken as the mean values of the initial ensembles. All other parameters and settings were same as in section 5.1.1. Using nobs $=2$, the EnKF assimilation is executed for $10 \mathrm{~s}$ and the evolution of estimated Youngs modulus with their uncertainty (standard deviations) is shown in Fig. 8a for three different initial values, including for $E=0.5 \mathrm{MPa}(+100 \%$ error $)$. The algorithm allows retrieving the target value independently on the initial guess. Table 4 compares the initial and final estimates of the Youngs's modulus with their associated uncertainties.

TABLE 4. Sensitivity with different initial values: final estimates of Youngs's modulus with their associated uncertainties. All values are in $\mathrm{MPa}$

\begin{tabular}{|c|c|c|}
\hline Initial guess of $E$ & Final estimate of $E$ & Uncertainty ( \pm standard deviation) \\
\hline 0.15 & 0.2367 & 0.0228 \\
\hline 0.35 & 0.2370 & 0.0250 \\
\hline 0.50 & 0.2450 & 0.0245 \\
\hline
\end{tabular}

(ii) The level of observation perturbation: As in section 2.1, the observations are perturbed by Gaussian noises. The noises represents possible errors in the measurement. For unbiased observations, perturbed observations are created by adding noise $\left(\sim\left(\mathcal{N}\left(0, \sigma^{2}\right)\right)\right.$, to the observation values; $\sigma$ represents the standard deviation. In test case $1, \sigma$ equals $5 \%$ of observation values and for the analysis, we chose two more levels of observation perturbations with $\sigma$ being $1 \%$ and $10 \%$ of observations values respectively. Using nobs $=2$, we perform the estimation procedure for $10 \mathrm{~s}$. The estimated Young's modulus with their uncertainty (standard deviations) are shown in Fig. 8b for the three different levels of observation perturbations. With different values of $\sigma$, the estimated $E$ 's converge to the target value, but with a slightly different rate. With a lower $\sigma$ (at $1 \%$ of observation values), the convergence rate is a little slower compared to the other two $\sigma$ 's, which was 
not anticipated. Table 5 compares the initial and final estimates of the Youngs's modulus with their uncertainties for the different level of perturbations used.

TABLE 5. Sensitivity with different level of observation perturbation: The initial guess of $E=0.5 \mathrm{MPa}$ and the final estimates of Youngs's modulus with their associated uncertainties are shown below. Perturbed observations are created by adding noise $\left(\sim\left(\mathcal{N}\left(0, \sigma^{2}\right)\right)\right.$, to the observation values. All values of $E$ and standard deviation are in MPa.

\begin{tabular}{|c|c|c|}
\hline$\sigma$ & Final estimate of $E$ & Uncertainty $( \pm$ standard deviation $)$ \\
\hline $1 \%$ of observation values & 0.2556 & 0.0122 \\
\hline $5 \%$ of observation values & 0.2450 & 0.0245 \\
\hline $10 \%$ of observation values & 0.2441 & 0.0398 \\
\hline
\end{tabular}

(iii) Bias in the known parameter values: In test case 1, we estimated the Young's modulus for artery \#vii assuming that we know the values of $E$ for all other arteries. We also assumed that all reflection coefficients were known. The performance of the estimation algorithm was tested by introducing biases in the known values of $E$ and $R_{t}$. Three different test were carried out as follows: (i) we randomly perturbed the values of reflection coefficients, $R_{t}$, with Gaussian noises having mean zero and a standard deviation at $5 \%$ of the values of $R_{t}$, (ii) the known values of Young's modulus, $E$ are randomly perturbed with Gaussian noises having mean zero and a standard deviation at $10 \%$ of the values of $E$ and (iii) known values of both reflection coefficients and Young's modulus are randomly perturbed with Gaussian noises having mean zero and a standard deviation at 5\% of the values of $R_{t}$ and $10 \%$ of the values of $E$. Pressure values in space were used as observations with the level of observation perturbation set at $5 \%$ of observation values and the estimation procedure is executed for $10 \mathrm{~s}$. For all tests, the initial value of $E$ assumes an error of $100 \%$. The evolution of the estimated Young's modulus with their uncertainties for three different tests is shown in Fig. $8 \mathrm{c}$ together with the evolution of estimated $E$ when known Young's modulus and reflection coefficients are unbiased. For all the 
cases, the estimated values converge, but they slightly deviate from the target value as reported in Table 6 which also gives indications of the level of uncertainties in these inversions.

TABLE 6. Sensitivity test: bias in the known parameter values. The initial guess of $E=0.5 \mathrm{MPa}$ and the final estimates of Youngs's modulus with their associated uncertainties are shown. All values of $E$ and standard deviation are in MPa.

\begin{tabular}{|l|c|c|}
\hline $\begin{array}{l}\text { Random perturbation of known } \\
\text { values of parameters by adding } \\
\text { noise }\left(\sim\left(\mathcal{N}\left(0, \sigma^{2}\right)\right) .\right.\end{array}$ & Final estimate of $E$ & Uncertainty ( \pm standard deviation) \\
\hline $\begin{array}{l}\text { unperturbed known parameters } \\
\text { values }\end{array}$ & 0.2450 & 0.0245 \\
\hline$\sigma$ is $5 \%$ of $R_{t}$ values & 0.2580 & 0.0132 \\
\hline$\sigma$ is $10 \%$ of $E$ values & 0.2752 & 0.0129 \\
\hline $\begin{array}{l}\sigma \text { is } 5 \% \text { of } R_{t} \text { values and } 10 \% \text { of } \\
E \text { values }\end{array}$ & 0.2480 & 0.0161 \\
\hline
\end{tabular}

(iii) Observation type: In inverse hemodynamics problems, observations such as blood pressure, cross section blood flow rates, artery wall movements or crosssection flow velocity can be made available. In test case 1 , the observations are pressure values histories at some specific locations in space. The behaviour of the estimation algorithm with different kinds of observations. To this end, we consider the flow rate in space as observations instead of the pressure. We perform the estimation procedure for $10 \mathrm{~s}$ using nobs $=2$ with the level of observation perturbation set at $5 \%$ of the observed values. We compare these results to those obtained with the pressure as observation. The evolution of estimated Youngs modulus with the associated uncertainties is shown in Fig. 8d. With both types of observations, the estimated Young's modulus converges to the target value with relatively small errors $(-1.95 \%$ and $+2.24 \%$ with pressure and flow rate as observations respectively). However, when the observations are based on the flow rates, the convergence is slightly faster, 
at least in this particular case. The final estimates of $E$ are $0.2450 \pm 0.0245 \mathrm{MPa}$ with pressure as observations and $0.2556 \pm 0.0059 \mathrm{MPa}$ with flow rate as observations.

5.1.3. Test case 2: The second test case deals with the estimation of the Young's modulus of all the arteries (i-ix) and the reflection coefficient at all the outlet boundaries (ii,iv,vi,vii,ix). All arteries are assumed to have the identical stiffness and thus, a Young's modulus of $E=0.2 \mathrm{MPa}$ is assigned to all. A reflection coefficient of $R_{t}=$ 0.6 , is assigned at all terminal vessels. The viscoelastic coefficient $\eta$ and the nonlinearity coefficient $\epsilon_{p}$ of the vessels are set to $0.115 \mathrm{kPa} . \mathrm{s}$ and 0 respectively for the forward simulation. The rest of the parameters are as in section 5.1.1. For the estimation problem, the Young's modulus of the arteries and the reflection coefficient at terminal arteries are sought using various numbers of observations. The ensemble size $q$ and the error covariance matrix $\mathbf{R}$ are as in section 5.1.1. The mean values for the initial guess of the Young's modulus and the reflection coefficient are set to $E=0.4 \mathrm{MPa}$ and $R_{t}=0.8$. The EnKF assimilation is executed for $12 \mathrm{~s}$, and the evolution of estimated $E$ and $R_{t}$ is shown in Figs. 9a and 9b respectively, for the different numbers of observations. The estimation procedure was able to identify the parameters with different nobs, though the convergence rate was much slower with $n o b s=1$. From these evolutions, we see that even if nobs $=1$ is enough to recover the values of $E$ and $R_{t}$, one should attempt to at least have two observations for faster convergence. The Young's modulus also appears to be simpler to identify than the reflection coefficient. This is possibly because the time required to propagate the information contained in any boundary condition throughout the whole domain is of order $L / c$ where $L$ is the size of the network and $c$ the wave speed. Instead, the Youngs's modulus directly impacts the wave speed so that it requires only $L s / c$ 
to feel any change in $E$, where $L s<L$ is the distance between two consecutive observation location. Table 7 shows the value of estimated Youngs's modulus and the reflection coefficients with errors (percentage deviation from target value) using different number of observations. In Fig. 9c, the pressure solution obtained with the estimated parameters using nobs $=2$, at the first observation point on left carotid is compared with the target and initial pressure profiles. The simulated and the target pressure waveforms have very similar shape with an error of less than $0.2 \%$ in the maximum pressure.

The next configuration involves a more realistic situation with available experimental data.

TABLE 7. Test case2: Estimated Youngs's modulus and reflection coefficients with errors (percentage deviation from target values) using different numbers of observations

\begin{tabular}{|l|c|c|c|c|}
\hline & Estimated $E(\mathrm{MPa})$ & Estimated $R_{t}$ & \% deviation from target $E$ & \% from target $R_{t}$ \\
\hline nobs $=1$ & 0.2064 & 0.5995 & 3.20 & -0.08 \\
\hline nobs $=2$ & 0.1993 & 0.6013 & -0.35 & 0.22 \\
\hline nobs $=3$ & 0.1988 & 0.6030 & -0.60 & 0.50 \\
\hline nobs $=4$ & 0.2006 & 0.5973 & 0.30 & -0.45 \\
\hline nobs $=5$ & 0.1992 & 0.5994 & -0.40 & -0.10 \\
\hline nobs $=6$ & 0.2003 & 0.5994 & 0.15 & -0.10 \\
\hline
\end{tabular}

5.2. A test case with experimental data. The efficiency of parameter estimation using the EnKF is presented where experimental data is used as the observations. We refer to the experiment in [24], where a simple human arterial network (see Fig. 4) with four bifurcations was designed using polymer tubes $(E=0.185 \mathrm{MPa})$ to validate the applicability of the blood flow model as presented in [24]. The tubes are filled with water, and to realize the reflection coefficients of approximately 0.5 , silicone tubes are connected at the end of the tubes to act as virtual peripheral sites. A pulse flow with the profile of half a cycle of a sinusoidal wave is used as input from 
the pump. The period of the pulse is $0.3 \mathrm{~s}$ with the total flow volume of $4.5 \mathrm{ml}$. The pressure waves propagating in the viscoelastic tubes were experimentally measured using a pressure sensor at $150 \mathrm{~mm}$ from the second bifurcation, which in an actual human body roughly corresponds to the carotid artery of the neck [24].

For this test case, we do not know if the solution of the inverse problem exists as the target has not been generated with the blood flow code. The aim is then to determine the best estimate of the Young's modulus $(E)$, reflection coefficient $\left(R_{t}\right)$ and viscoelastic coefficient $(\eta)$ from the values of the experimentally measured pressure, which are taken as observations for the inverse problem. It is assumed that $E$ and $\eta$ are identical for all tubes and $R_{t}$ is same at each terminal tube. The ensemble size $q=20$ and the error covariance matrix $\mathbf{R}$ are defined as in section 5.1.1. The pressure measurements are only available at one point on the carotid artery. The frequency of data assimilation is $0.01 \mathrm{~s}$.

To test the sensitivity to initial parameter values, we investigate the performance of the EnKF parameter estimation algorithm using three different sets of initial guess for the three parameters $\left(E, R_{t}\right.$ and $\left.\eta\right)$. The mean values for the initial guess of the parameters were: $\left(E(M P a), R_{t}, \eta(K P a \cdot s)\right) \in\{(0.2,0.6,0.3),(0.6,0.3,0.4)\},(0.4,0.8,0.5)$. The estimated parameter values do not change significantly after $16 \mathrm{~s}$ of EnKF assimilation as shown in Fig. 10. From Fig 10, we see that the different guesses for initial mean values of the parameters seem not to have a significant impact on the converged assimilated result. The initial guess of the parameters and their best estimates obtained with their associated uncertainty (standard deviation) with three different initial guesses using the EnKF are shown in Table 8. 
TABLE 8. Sensitivity to initial parameter values for the test with experimental data. The initial guess of the parameters and their best EnKF estimates with their associated uncertainty (standard deviation).

\begin{tabular}{|c|c|c|c|}
\hline Parameter & Initial guess & EnKF estimate & Error ( \pm standard deviation $)$ \\
\hline \multirow{3}{*}{$E(\mathrm{MPa})$} & 0.2 & 0.1111 & 0.0024 \\
\cline { 2 - 4 } & 0.6 & 0.1226 & 0.0025 \\
\cline { 2 - 4 } & 0.4 & 0.1150 & 0.0028 \\
\hline \multirow{3}{*}{$R_{t}$} & 0.6 & 0.5199 & 0.0033 \\
\cline { 2 - 4 } & 0.3 & 0.5146 & 0.0075 \\
\hline \multirow{3}{*}{$\eta(\mathrm{KPa} \cdot \mathrm{s})$} & 0.8 & 0.5290 & 0.0048 \\
\cline { 2 - 4 } & 0.3 & 0.3710 & 0.0124 \\
\cline { 2 - 4 } & 0.4 & 0.3760 & 0.0104 \\
\hline
\end{tabular}

The blood flow model is then used with the estimated parameters to obtain the pressure profile at the observation point. Figure 11 shows the comparison between the pressure profile obtained with the three sets of estimated parameters, the numerical pressure profiles from the 1D blood flow model as reported in [24], the measured pressure waves obtained from the experiment and the pressure profile obtained from the three different sets of initial parameters. The simulated waveforms are similar to the target pressure waveform. We compare the systolic (maximum) pressure between the target (experimental pressure waveform) and the simulated pressures obtained from different sets of estimated parameters. The difference is shown in Table 9. In all the cases, the error is less than $2 \%$. The results demonstrate that even with nobs $=1$, the Young's modulus, reflection coefficient, and the viscoelastic coefficient can be estimated with good accuracy using the proposed method. 
TABle 9. Test case3: Comparison of the systolic (maximum) pressure between the target (experimental pressure waveform) and the simulated pressures obtained from different sets of estimated parameters. The target systolic pressure is $4.02 \mathrm{MPa}$

\begin{tabular}{|c|c|c|}
\hline Estimated parameter set $\left\{E(M P a), R_{t}, \eta(k P a . s)\right\}$ & maximum pressure (MPa) & \% error \\
\hline set 1 $(0.1111,0.5199,0.371)$ & 4.01 & -0.24 \\
\hline set 2 $(0.1226,0.5146,0.376)$ & 4.08 & 1.50 \\
\hline set $3(0.1150,0.529,0.381)$ & 4.04 & 0.51 \\
\hline
\end{tabular}

\section{Discussions}

In this paper, we have demonstrated the applicability of EnKF to estimate the Young's modulus, reflection coefficient, and viscoelastic coefficient. A similar approach can also be used to estimate other hemodynamics parameters such as resistance and compliance in a Windkessel model. The EnKF and thus the estimation algorithm provides the estimates of poorly known parameter values with their uncertainties. Sensitivity analysis with respect to the initial guess of parameters, the level of observation perturbation, the effect of bias in known parameter values and the type of observations is carried out. Further analysis can be done on the efficiency of the estimation algorithm with respect to the locations of available observations. We need to make sure the size of the ensemble is correctly chosen. One also sees that very few spatial observation are necessary as the approach performs even with solely one spatial observation point. We also discussed the robustness of the inversion for different types of target observations (pressure or flow rate). We have shown a method of choosing an ensemble size using RMSE, but the efficiency of the EnKF parameter estimation algorithm may depend on other factors such as level of observation perturbation, the location of the observations, their types and also on the type of parameters to be estimated. The approach needs to be seen as a help to diagnosis tool and not a definite opinion. We mentioned that one issue is that 
uniqueness is not guaranteed. This might, therefore, impact clinical applications. Indeed, an incorrect Youngs modulus might be obtained still providing a nice model fit and obviously, this might mislead the clinician. The approach, therefore, needs to be adopted in a Bayesian procedure with a priori information on the admissibility of the outcome by the clinicians and the outcome should definitely not been considered as a final opinion.

We aim at having an approach with moderate complexity to describe the physics of the problem and which is usable in practice. This is why any forward model based on a multi-dimensional flow model is out of the table. Other works, for instance, present data assimilation together with three-dimensional flow models based on fully 3D Navier-Stokes [18], which require heavy computational effort in addition to an increase in the complexity of the inverse problem. These approaches also require good know-how by the user and substantial learning efforts. We use a reduction in dimension to bring the cost of one state evaluation to the order of a minute on standard computers available in clinics. Then natural parallelism in EnKF makes a time to solution of the order of the number of EnKF iterations in minutes, which in the present case leads to approximately two hours. Still this can be considered too costly and our current effort is to reduce complexity even further.

\section{Study Limitations}

The first limitation of our current study is concerned with the size of the arterial network being adopted. We used a network consisting of 9 vessels, and the efficiency of the proposed estimation algorithm has to be tested for a larger arterial network, including complex network such as the circle of Willis in the cerebral vasculature. 
Secondly, an ensemble size of 20 is taken as an optimal size for the parameter estimation in the current study. An effect of taking a larger sample size on the estimation procedure can also be studied. The efficiency and convergence rate also depends on the level of observation perturbation, and another limitation is to identify the optimal level. It is also important to investigate the maximum number of parameters that can be estimated for a given arterial network with a given number of measurements available. In the current study, we adopted 1D blood flow model with a constant resistance boundary condition. As discussed before, there is no real limitation regarding the boundary description and thus, the estimation algorithm can be applied to a blood flow model coupled to a Windkessel model.

\section{Conclusion}

A parameter estimation technique to compute the uncertain elastic and the terminal properties of networks of 1D blood vessels using the Ensemble Kalman filter has been studied. The tests have been limited to the estimation of elastic moduli (Young's modulus) of the network, the reflection coefficient at the terminal vessels and the viscoelastic coefficient. The results confirm that the method is quite robust and permits to recover the arteries stiffness in a reasonable amount of time consistent with patient observation time at the hospital. Except with the computation of the Kalman gain, all the operations on the ensemble members are independent. This implies that their parallelization can be trivially carried out, thus decreasing the computational time needed to solve the inverse hemodynamics problem. The time to solution for this simulation is about 30 minutes on a parallel computer with 20 cores, which is basically one node of current standard distributions. The model 
simulations performed with the estimated parameter values produced accurate pressure profiles, which followed closely with the target profiles showing the effectiveness and the efficiency of both the estimation algorithm and the blood flow model. Also it has been shown that the approach is effective with only a few observations, well suited to real clinical applications.

\section{Acknowledgements}

Research done under the European Union Framework Programme Erasmus Mundus KITE (2013-2617 / 001-001 - EMA2)

\section{REFERENCES}

[1] Benetos A, Waeber B, Izzo J, Mitchell G, Resnick L, Asmar R, Safar M. Influence of age, risk factors, and cardiovascular and renal disease on arterial stiffness: clinical applications. American journal of hypertension 2002; 15(12):11011108.

[2] Messas E, Pernot M, Couade M. Arterial wall elasticity: state of the art and future prospects. Diagnostic and interventional imaging 2013; 94(5):561-569.

[3] Lorenc AC. Atmospheric data assimilation. Meteorological Office, 1995.

[4] Lahoz W, Khattatov B, Menard R. Data assimilation: making sense of observations. Springer Science \& Business Media, 2010.

[5] Evensen G. The ensemble kalman filter for combined state and parameter estimation. Control Systems, IEEE 2009; 29(3):83-104.

[6] Annan J, Hargreaves J, Edwards N, Marsh R. Parameter estimation in an intermediate complexity earth system model using an ensemble kalman filter. Ocean modelling 2005; 8(1):135-154. 
[7] Anderson JL. An ensemble adjustment kalman filter for data assimilation. Monthly weather review 2001; 129(12):2884-2903.

[8] Gillijns S, De Moor B. Model error estimation in ensemble data assimilation. Nonlinear Processes in Geophysics 2007; 14(1):59-71.

[9] Baek SJ, Hunt BR, Kalnay E, Ott E, Szunyogh I. Local ensemble kalman filtering in the presence of model bias. Tellus A 2006; 58(3):293-306.

[10] Yang X, Delsole T. Using the ensemble kalman filter to estimate multiplicative model parameters. Tellus A 2009; 61(5):601-609.

[11] Liu J, West M. Combined parameter and state estimation in simulation-based filtering. Sequential Monte Carlo methods in practice. Springer, 2001; 197-223.

[12] Srikanthan R, Amirthanathan G, Kuczera G. Real-time flood forecasting using ensemble kalman filter. MODSIM 2007 International Congress on Modelling and Simulation. Modelling and Simulation Society of Australia and New Zealand, 2007; 1789-1795.

[13] Zupanski D, Zupanski M. Model error estimation employing an ensemble data assimilation approach. Monthly Weather Review 2006; 134(5):1337-1354.

[14] Godinez HC, Reisner JM, Fierro AO, Guimond SR, Kao J. Determining key model parameters of rapidly intensifying hurricane guillermo (1997) using the ensemble kalman filter. arXiv preprint arXiv:110\%.440 2011; .

[15] Massonnet F, Goosse H, Fichefet T, Counillon F. Calibration of sea ice dynamic parameters in an ocean-sea ice model using an ensemble kalman filter. Journal of Geophysical Research: Oceans 2014; 119(7):4168-4184.

[16] Lombardi D. Inverse problems in 1d hemodynamics on systemic networks: A sequential approach. International journal for numerical methods in biomedical 
engineering 2014; 30(2):160-179.

[17] Moireau P, Bertoglio C, Xiao N, Figueroa CA, Taylor C, Chapelle D, Gerbeau JF. Sequential identification of boundary support parameters in a fluidstructure vascular model using patient image data. Biomechanics and modeling in mechanobiology 2013; 12(3):475-496.

[18] Pant S, Fabrèges B, Gerbeau JF, Vignon-Clementel I. A methodological paradigm for patient-specific multi-scale cfd simulations: from clinical measurements to parameter estimates for individual analysis. International journal for numerical methods in biomedical engineering 2014; 30(12):1614-1648.

[19] Bertoglio C, Moireau P, Gerbeau JF. Sequential parameter estimation for fluidstructure problems: Application to hemodynamics. International Journal for Numerical Methods in Biomedical Engineering 2012; 28(4):434-455.

[20] Chabiniok R, Moireau P, Lesault PF, Rahmouni A, Deux JF, Chapelle D. Estimation of tissue contractility from cardiac cine-mri using a biomechanical heart model. Biomechanics and modeling in mechanobiology 2012; 11(5):609-630.

[21] Martin V, Clément F, Decoene A, Gerbeau JF. Parameter identification for a one-dimensional blood flow model. ESAIM: Proceedings, vol. 14, EDP Sciences, $2005 ; 174-200$.

[22] Spilker RL, Taylor CA. Tuning multidomain hemodynamic simulations to match physiological measurements. Annals of biomedical engineering 2010; 38(8):2635-2648.

[23] Lassila T, Manzoni A, Quarteroni A, Rozza G. A reduced computational and geometrical framework for inverse problems in hemodynamics. International journal for numerical methods in biomedical engineering 2013; 29(7):741-776. 
[24] Saito M, Ikenaga Y, Matsukawa M, Watanabe Y, Asada T, Lagrée PY. Onedimensional model for propagation of a pressure wave in a model of the human arterial network: Comparison of theoretical and experimental results. Journal of Biomechanical Engineering 2011; 133(12):121 005.

[25] Evensen G. Sequential data assimilation with a nonlinear quasi-geostrophic model using monte carlo methods to forecast error statistics. Journal of Geophysical Research: Oceans (1978-2012) 1994; 99(C5):10 143-10 162.

[26] Thomas SJ, Hacker J, Anderson J. A robust formulation of the ensemble kalman filter. Quarterly Journal of the Royal Meteorological Society 2009; 135(639):507-521.

[27] Sakov P, Oke PR. A deterministic formulation of the ensemble kalman filter: an alternative to ensemble square root filters. Tellus $A$ 2008; 60(2):361-371.

[28] Evensen G. The ensemble kalman filter: Theoretical formulation and practical implementation. Ocean dynamics 2003; 53(4):343-367.

[29] Houtekamer PL, Mitchell HL. Data assimilation using an ensemble kalman filter technique. Monthly Weather Review 1998; 126(3):796-811.

[30] Houtekamer P, Mitchell HL. Ensemble kalman filtering. Quarterly Journal of the Royal Meteorological Society 2005; 131(613):3269-3289.

[31] Hamill TM, Snyder C. A hybrid ensemble kalman filter-3d variational analysis scheme. Monthly Weather Review 2000; 128(8):2905-2919.

[32] Bishop CH, Etherton BJ, Majumdar SJ. Adaptive sampling with the ensemble transform kalman filter. part i: Theoretical aspects. Monthly weather review $2001 ; \mathbf{1 2 9}(3): 420-436$. 
[33] Whitaker JS, Hamill TM. Ensemble data assimilation without perturbed observations. Monthly Weather Review 2002; 130(7):1913-1924.

[34] Ott E, Hunt BR, Szunyogh I, Zimin AV, Kostelich EJ, Corazza M, Kalnay E, Patil D, Yorke JA. A local ensemble kalman filter for atmospheric data assimilation. Tellus A 2004; 56(5):415-428.

[35] Gillijns S, Mendoza OB, Chandrasekar J, De Moor B, Bernstein D, Ridley A. What is the ensemble kalman filter and how well does it work? American Control Conference, 2006, IEEE, 2006.

[36] Shen Z, Tang Y. A modified ensemble kalman particle filter for non-gaussian systems with nonlinear measurement functions. Journal of Advances in Modeling Earth Systems 2015; .

[37] Tang Y, Ambandan J, Chen D. Nonlinear measurement function in the ensemble kalman filter. Advances in Atmospheric Sciences 2014; 31(3):551-558.

[38] Mohammadi B. Ensemble kalman filters and geometric characterization of sensitivity spaces for uncertainty quantification in optimization. Computer Methods in Applied Mechanics and Engineering 2015; 290:228-249.

[39] Burgers G, Jan van Leeuwen P, Evensen G. Analysis scheme in the ensemble kalman filter. Monthly weather review 1998; 126(6):1719-1724.

[40] Houtekamer PL, Mitchell HL. A sequential ensemble kalman filter for atmospheric data assimilation. Monthly Weather Review 2001; 129(1):123-137.

[41] Ambadan JT, Tang Y. Sigma-point kalman filter data assimilation methods for strongly nonlinear systems. Journal of the Atmospheric Sciences 2009; 66(2):261-285. 
[42] Elsheikh AH, Pain C, Fang F, Gomes J, Navon I. Parameter estimation of subsurface flow models using iterative regularized ensemble kalman filter. Stochastic environmental research and risk assessment 2013; 27(4):877-897.

[43] Sherwin S, Formaggia L, Peiro J, Franke V. Computational modelling of 1d blood flow with variable mechanical properties and its application to the simulation of wave propagation in the human arterial system. International Journal for Numerical Methods in Fluids 2003; 43(6-7):673-700.

[44] Wang X, Fullana JM, Lagrée PY. Verification and comparison of four numerical schemes for a $1 \mathrm{~d}$ viscoelastic blood flow model. Computer methods in biomechanics and biomedical engineering 2015; 18(15):1704-1725.

[45] Peiró J, Veneziani A. Reduced models of the cardiovascular system. Cardiovascular mathematics. Springer, 2009; 347-394.

[46] Mynard J, Nithiarasu P. A 1d arterial blood flow model incorporating ventricular pressure, aortic valve and regional coronary flow using the locally conservative galerkin (lcg) method. Communications in Numerical Methods in Engineering 2008; 24(5):367-417.

[47] Blanco P, Watanabe S, Feijoo R. Identification of vascular territory resistances in one-dimensional hemodynamics simulations. Journal of biomechanics 2012; 45(12):2066-2073.

[48] Quarteroni A, Formaggia L. Mathematical modelling and numerical simulation of the cardiovascular system. Computational Models for the Human Body, Handbook of Numerical Analysis, vol. 12. Elsevier, 2004; 3 - 127, doi: http://dx.doi.org/10.1016/S1570-8659(03)12001-7. 
[49] Formaggia L, Lamponi D, Tuveri M, Veneziani A. Numerical modeling of 1d arterial networks coupled with a lumped parameters description of the heart. Computer methods in biomechanics and biomedical engineering 2006; 9(5):273288.

[50] Marchandise E, Willemet M, Lacroix V. A numerical hemodynamic tool for predictive vascular surgery. Medical engineering \& physics 2009; 31(1):131-144.

[51] Formaggia L, Quarteroni A, Veneziani A. Cardiovascular Mathematics: Modeling and simulation of the circulatory system, vol. 1. Springer Science \& Business Media, 2010.

[52] Reymond P, Merenda F, Perren F, Rüfenacht D, Stergiopulos N. Validation of a one-dimensional model of the systemic arterial tree. American Journal of Physiology-Heart and Circulatory Physiology 2009; 297(1):H208-H222.

[53] Boileau E, Nithiarasu P, Blanco PJ, Müller LO, Fossan FE, Hellevik LR, Donders WP, Huberts W, Willemet M, Alastruey J. A benchmark study of numerical schemes for one-dimensional arterial blood flow modelling. International journal for numerical methods in biomedical engineering 2015; 31(10).

[54] Formaggia L, Lamponi D, Quarteroni A. One-dimensional models for blood flow in arteries. Journal of engineering mathematics 2003; 47(3-4):251-276.

[55] Vignon IE, Taylor CA. Outflow boundary conditions for one-dimensional finite element modeling of blood flow and pressure waves in arteries. Wave Motion $2004 ; 39(4): 361-374$.

[56] Du T HD, Cai D. Outflow boundary conditions for blood flow in arterial trees. PLoS ONE 2015; 10(5):e0128597. 
[57] Alastruey J, Parker K, Peiró J, Sherwin S. Lumped parameter outflow models for 1-d blood flow simulations: effect on pulse waves and parameter estimation. Communications in Computational Physics 2008; 4(2):317-336. 
Evolution of estimated $E$ with different initial guess

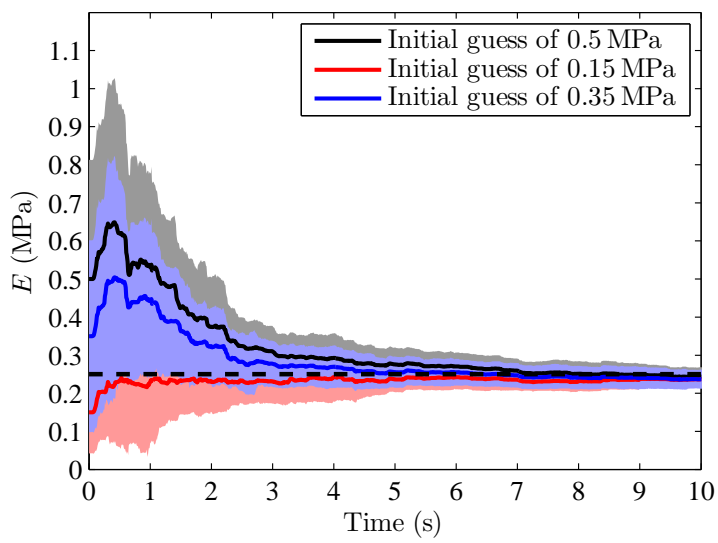

(A)

Estimated $E$ with different level of observation perturbations

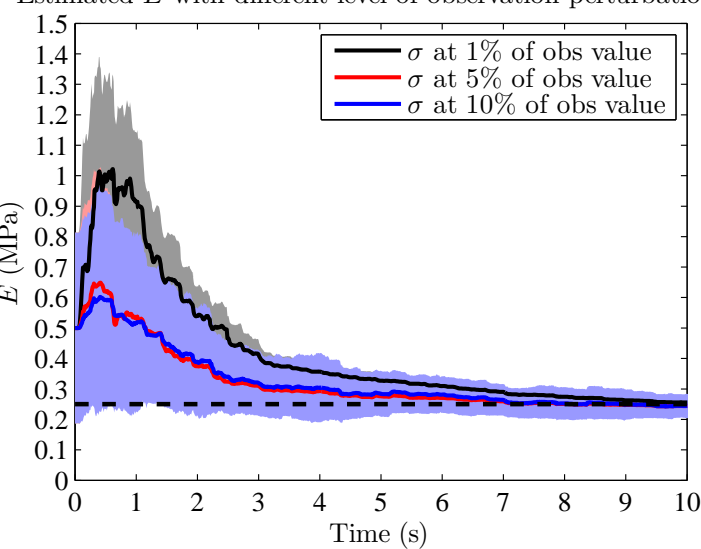

(B)

Evolution of estimated $E$ with biases in known parameters

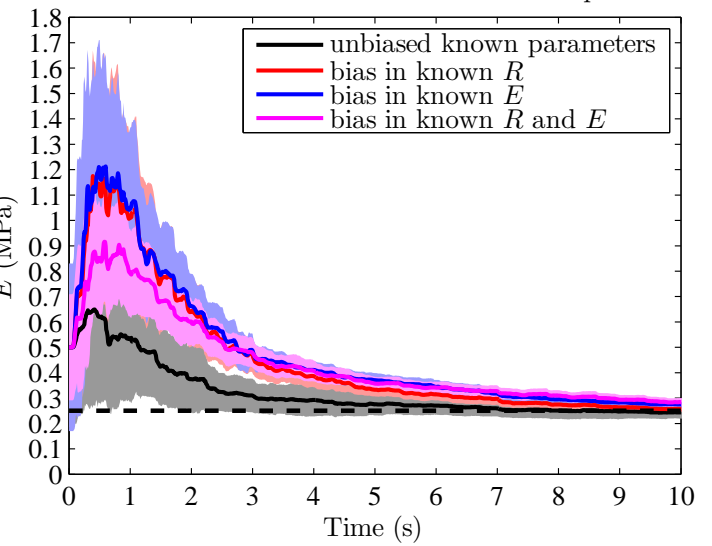

(C)

Evolution of estimated $E$ with different observation type

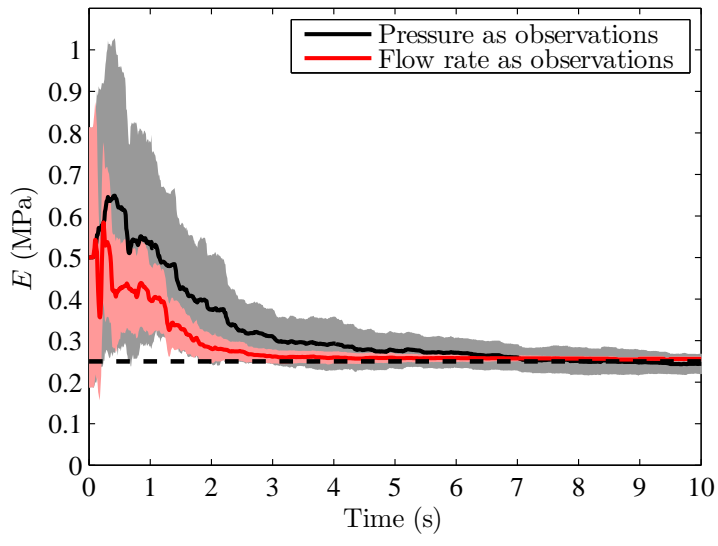




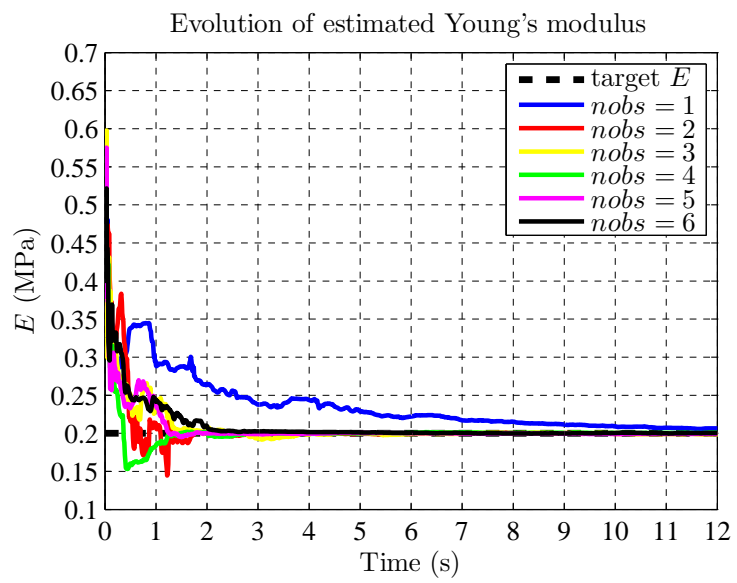

(A)

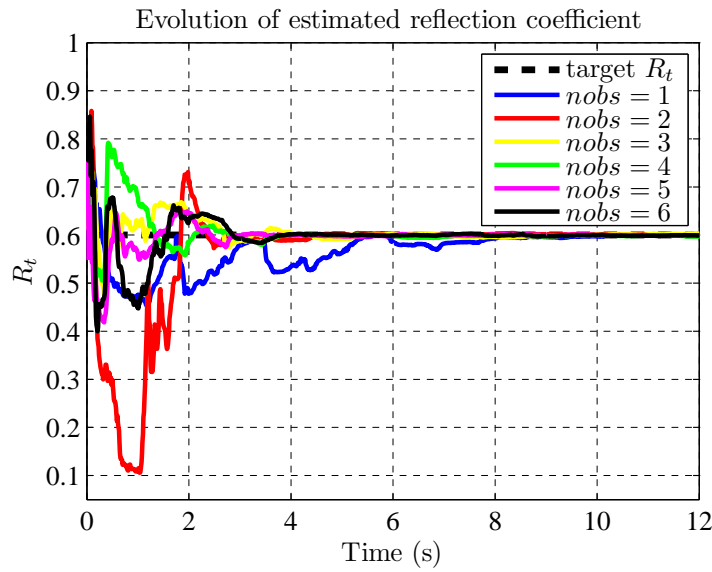

(B)

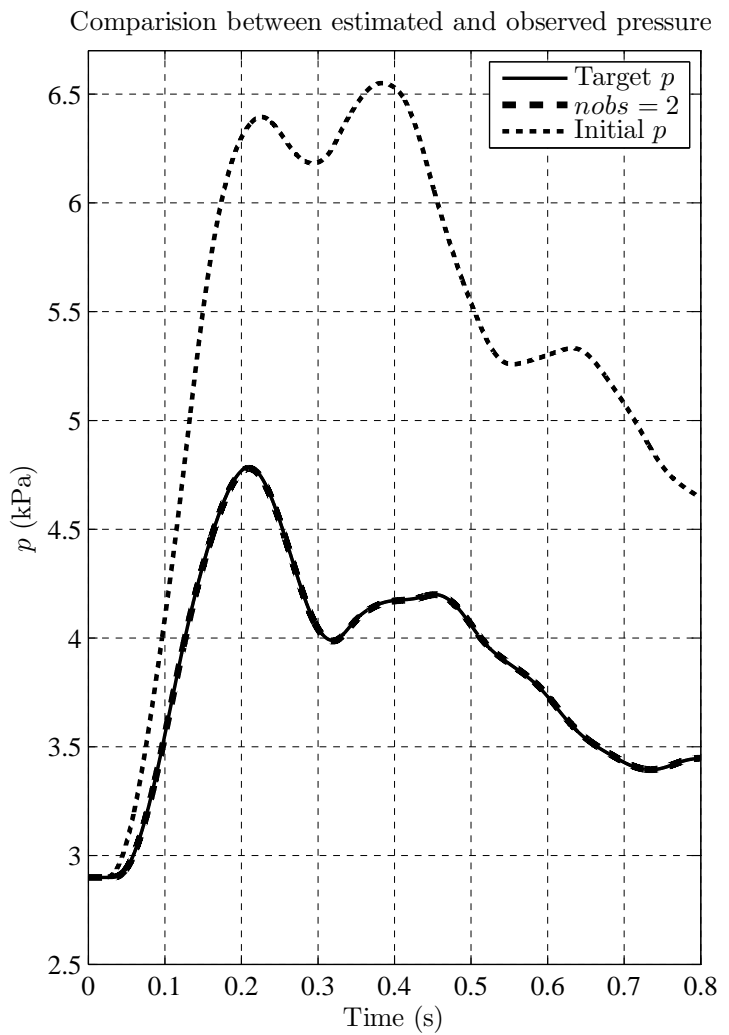

(C)

Figure 9. (9a) shows the evolution of the Young's modulus using a different number of observations for case 2 . The initial value is $0.4 \mathrm{MPa}$ and the target is $0.2 \mathrm{MPa}$. (9b) shows the evolution of the 


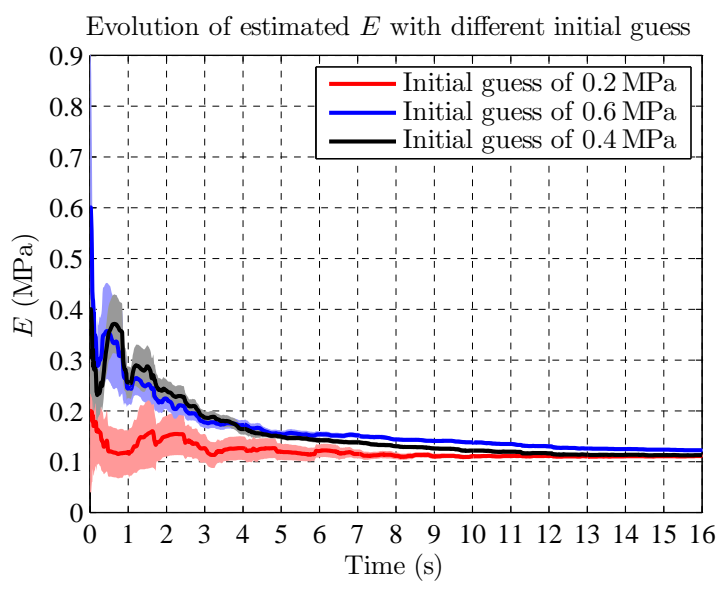

(A)

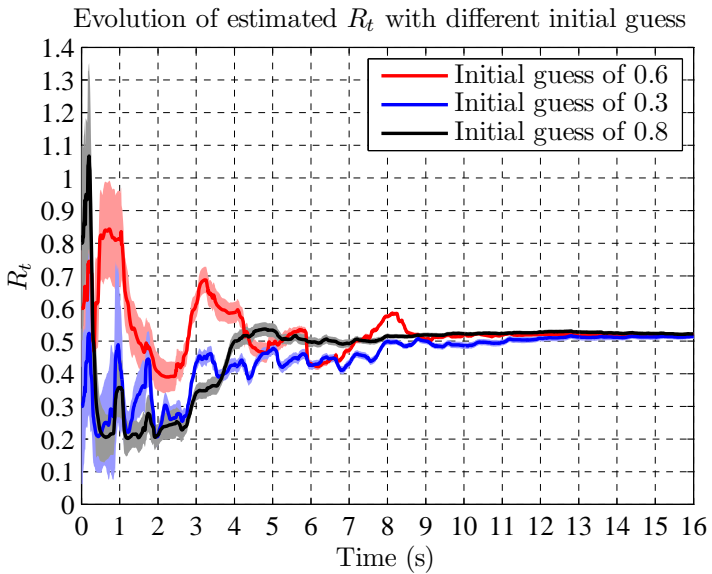

(B)

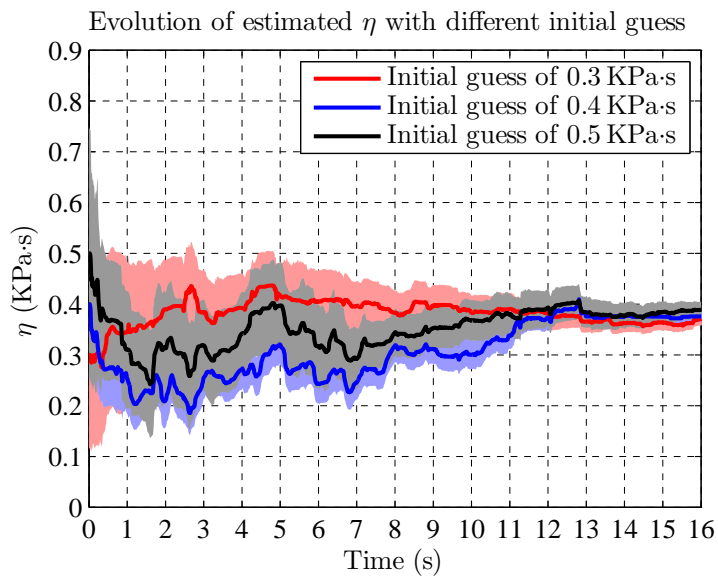

(C)

Figure 10. Sensitivity of EnKF parameter estimation to different sets of initial parameter values. 10a-10c show the evolution of Young's modulus, reflection coefficient, and viscoelastic coefficient respectively for the test case using the experimental data with different sets of initial values. The set of initial guess of the parameters, $\left\{E(M P a), R_{t}, \eta(k P a . s)\right\}$ are: in RED $\{0.2,0.6,0.3\}$, in BLUE $\{0.6,0.3,0.4\}$, in BLACK $\{0.4,0.8,0.5\}$. The shaded areas represent one standard deviation around the mean values (solid lines). 


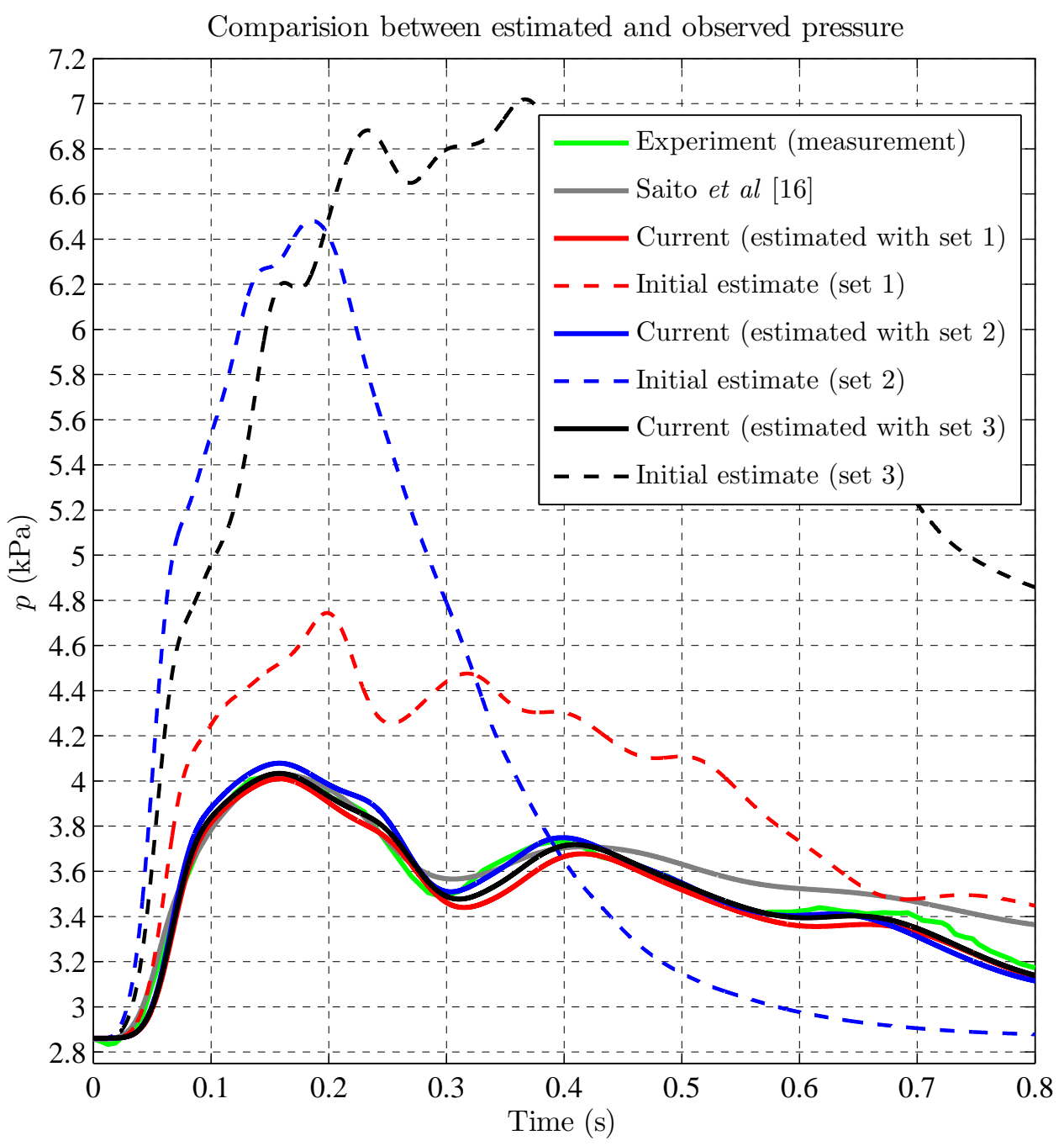

Figure 11. Comparison between the pressure profile obtained with the three sets of estimated parameters, the numerical pressure profiles from the 1D blood flow model as reported in [24], the measured pressure waves obtained from the experiment and the pressure profile obtained from the three different sets of initial parameters. The dashed lines are the initial pressure waveforms and the solid lines are the ones obtained from the estimated parameters. The set of initial guess of the parameters, $\left\{E(M P a), R_{t}, \eta(k P a . s)\right\}$ are: set $1\{0.2,0.6,0.3\}$, set $2\{0.6,0.3,0.4\}$ and set $3\{0.4,0.8,0.5\}$. 